\title{
THE ELECTRICAL CONDUCTIVITY OF NON- AQUEOUS SOLUTIONS ${ }^{\text {}}$
}

\section{BY AZARIAH T. IINCOLN}

A preliminary report on the electrical conductivity of nonaqueous solutions by Frofessor Kahlenberg and myself has appeared in an article entitled "The Dissociation Power of Solvents." = I have since been engaged in investigating the subject further, and desire in this present article to present the experimental results that I have obtained and also to inquire what the bearing of these facts is upon the theory of electrolytic dissociation as promulgated by Arrhenitts, and to determine to what extent this theory can be applied to non-aqueous solutions. In view of the fact that Arrhenins' theory is not accepted by a large number of chemists to-day, and in the face of the data that have been collected, one may well hesitate to apply this theory to nonaqueons solutions until there is a sufficiently firm experimental basis to justify it. Anxiliary theories have been promulgated to explain the facts presented by numerons investigators, and an attempt will be made to show to what extent these theories are compatible with the experimental results herein presented.

In an investigation of the electrical conductivity of nonaqueous solutions, the chief requirement is that both the solvents and the dissolved substances be absolutely free from water. To accomplish this is not an easy task. Then, too, the question arises as to what anhydrous salts are soluble in the various solrents. In many cases this could only be answered by experi-

'From the author's thesis for the degree of IDoctor of Philosophy, presented to the faculty of the University of Wisconsin, June, 1899. Published in full in the Transactions of the Wisconsin Academy of Sciences, Arts, and Letters, Vol. XII, pp. 395-453.

zJour. Phys. Chem. 3, I 2 (I 899 ). 
ment. The qualitative results previously published were confined to ferric chloride, antimony trichloride, bismuth trichloricle and mercuric chloride. In addition to these, salts of other heary metals have been employed in this investigation. Even when these salts were readily soluble, conducting solutions were not always obtained. In order to determine what solutions conduct and to estimate ronghly at least the relative magnitude of the conductivity, it was necessary to perform an elaborate series of qualitative experiments to ascertain what solvents yield solutions having a conductivity sufficient to justify quantitative measurements.

The method employed in making these tests has been prerionsly described. The solvents were all of the C. P. variety of standard makes. The salts employed were absolutely anhydrons and the method enployed in their preparation and dehydration will now be given. The preparation of cupric chloride, mercuric chloride, stannous and almminium chlorides, stannic chloride, arsenic trichloride, and the trichloride of phosphorus has been previonsly described. A C. P. sample of lithinm chloride from Merck was dehydrated for several days at a temperature not to exceed $120^{\circ}$. It was then removed to a mortar, thoronghly pulverized and then replaced in the air-bath for about a day longer. The following salts were recrystallized several times and then dehydrated in the manner just described: manganons chloride from Merck, chlorides of nickel and cadminum from Schnchardt, and cobaltic chloride from Trommsdorff. The C. P. samples of lead nitrate and mercuric iodide from Merck, mercuric cyanide from Trommsdorff, and zinc chloride from Kahlbaun, were dehydrated in the manner similar to that just described. The silver cyanide was prepared from potassium cyanicle and silver nitrate by Professor Kalilenberg, who dehydrater it and upon subsequent analysis found it to be pure.

Since the qualitative determinations throw some light upon the dissociative power of the solvents, the following additional results are given in Table I. In the first two columns are given 
the names and formulic of the solvents. In the next eighteen columns is indicated whether the solutions of the salts whose formula head the respective columns, conduct electricity. When the solution conducted so poorly that no deflection of the galvanometer needle was observed, the fact is indicated by the minus sign (-). The plus sign ( ) indicates that the solution did conduct. The addition of the interrogative sign to the plus sign (- ?) indicates that a very slight movement of the needle was detected; and whet the plus signi is followed by the exclanation mark $(-$ !), this indicates that the solution conducts sufficiently well to make a qualitative determination desirable. Blank spaces indicate that deterninations were not made, and the letter $i$ indicates that the salt was insoluble in the solvent or very difficultly soluble. In the last two columns respectively are given the dielectric constants and the coefficients of association of the solvents. The data in these two columns are as comnplete as conld be obtained from the literature. ${ }^{.}$The letters $\mathrm{K}$ and $S$ refer to the article by Ramsay and Shields" as authority. $R$ and $A$ to the work of Ramsay and Aston, ${ }^{3}$ and $\left.I\right)$ and $F$ to that of Dutoit and Friderich. ${ }^{*}$ The letter $V$ designates deter. minations by Volmer, C by Carrara, and S by Schlamp.

As many quantitative determinations of the conductivity of alcoholic solutions have been made, it was unnecessary to make Chem, 23,308 is 897 :

"Jour. Chem1. Soc. 63, rosg i 1893 .

"Ihic. 65, I6S Is9.t:

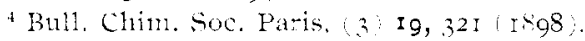

(I) Carrara. Gazz. Chim. Ital. 24, II. 504. (Ref.) Jour. Chem. Suc, 68, ii. 302 (1895): Ibid. 26, I, I Ig. (Ref, J Jour. Chemr. Soc. 70, ii, 5 I I (1896): Ibirl 27. I, 422. (Ref.) Jour, Chem. Soc. 72, ii, 473 IS97) (2) Cattaneo. Rencl

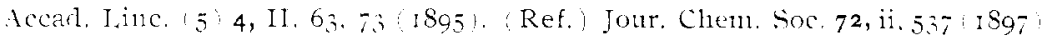

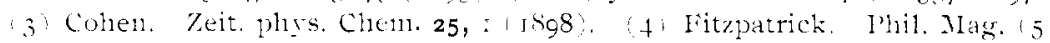

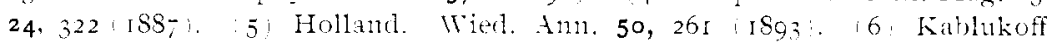
J. Russ. Cheml Soc. 23, 291 . Ref., Jour. Cheml. Soc. 64, ii, 151 I Is93 7 I'feiffer. Wied. Ann. 26, 3t 1885 ', is i Schall. \%eit. phys Chem. i4

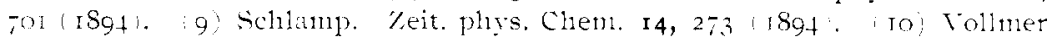
Wied. Ann, 52, 328 isgt: il Zelinsky and Krapiwin. Zeit phys. Chem. $21,35: 1896:$ 
460

- - - - -

Solvent

Formula

TABLE I
Hzariah T. Lincoln

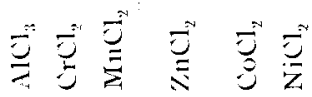

Methyl alcohol

Ethyl alcohol

Propyl alcohol

Allyl alcohol

Benzyl alcohol

Benzaldehycle

Salicylic aldehyde

Furfurol

Acetone

Methyl-propyl ketone

Acetophenone

Ethyl acetate

Ethyl monochloracetate

Ethyl cranacetate

Ethyl acetoacetate

Ethyl benzoate

Ethyl oxalate

Ethyl nitrate

Anyl nitrite

Nitrobenzene

o-Nitrotoluene

Aniline

Xylidine (meta astm.)

Benzonitrile

Pyridine

Piperidine

Quinoline

Phosphorus trichloride

Tin tetrachloride
$\mathrm{CH}_{3} \mathrm{OH}$

$\mathrm{C}_{2} \mathrm{H}_{5} \mathrm{OH}$

$\mathrm{CH}_{3}\left(\mathrm{CH}_{2}\right)_{2} \mathrm{OH}$

$\mathrm{C}_{3} \mathrm{H}_{5} \mathrm{OH}$

$\mathrm{C}_{2} \mathrm{H}_{3} \mathrm{CH}_{2} \mathrm{OH}$

$\mathrm{C}_{1} \mathrm{H}_{2}, \mathrm{COH}$

$\mathrm{C}_{4} \mathrm{H}_{4} \mathrm{CHO} . \mathrm{OH}$

$\mathrm{C}_{2} \mathrm{H}_{4} \mathrm{O}_{2}$

$\left(\mathrm{CH}_{3}\right)_{2} \mathrm{CO}$

$\mathrm{CH}_{3} \mathrm{C}_{3} \mathrm{H} . \mathrm{CO}$

$\mathrm{CH}_{3} \mathrm{COC}_{8} \mathrm{H}_{8}$

$\mathrm{CH}_{3} \mathrm{COOC}_{2} \mathrm{H}_{5}$

$\mathrm{CH}_{2} \mathrm{ClCOOC}_{2} \mathrm{H}$

$\mathrm{CH}_{2} \mathrm{CN} \cdot \mathrm{COOC}_{2} \mathrm{H}_{5}$

$\mathrm{CH}_{3} \mathrm{CO} \cdot \mathrm{CH}_{2} \mathrm{COOC}_{2} \mathrm{H}_{8}$

$\mathrm{C}_{8} \mathrm{H}_{3} \mathrm{COOC}_{2} \mathrm{H}_{3}$

$\left(\mathrm{COO} . \mathrm{C}_{8} \mathrm{H}_{0}\right)$.

$\mathrm{C}_{2} \mathrm{H}_{4} \mathrm{NO}_{3}$

$\mathrm{C}_{3} \mathrm{H}_{1}, \mathrm{NO}_{2}$

$\mathrm{C}_{4} \mathrm{H}_{4} \mathrm{NO}_{2}$

$\mathrm{C}_{4} \mathrm{H}_{4} \cdot \mathrm{CH}_{2} \mathrm{NO}$

$\mathrm{C}_{4} \mathrm{H}_{3} \mathrm{NH}_{4}$

$\mathrm{C}_{4} \mathrm{H}_{3}\left(\mathrm{CH}_{4}, \mathrm{NH}\right.$.

$\mathrm{C}_{\mathrm{C}} \mathrm{HCX}$

$\mathrm{C}_{2} \mathrm{H}_{3} \mathrm{~N}$

$\mathrm{C}_{5} \mathrm{H}_{11} \mathrm{~N}$

$\mathrm{C}_{2} \mathrm{H}_{7} \mathrm{~N}$

$\mathrm{PCl}_{3}$

$\mathrm{SnCl}_{+}$
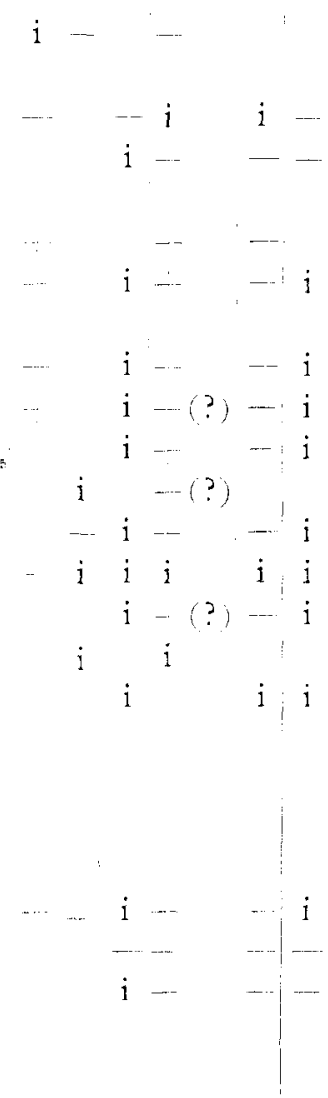
TABLE I-(Continued)

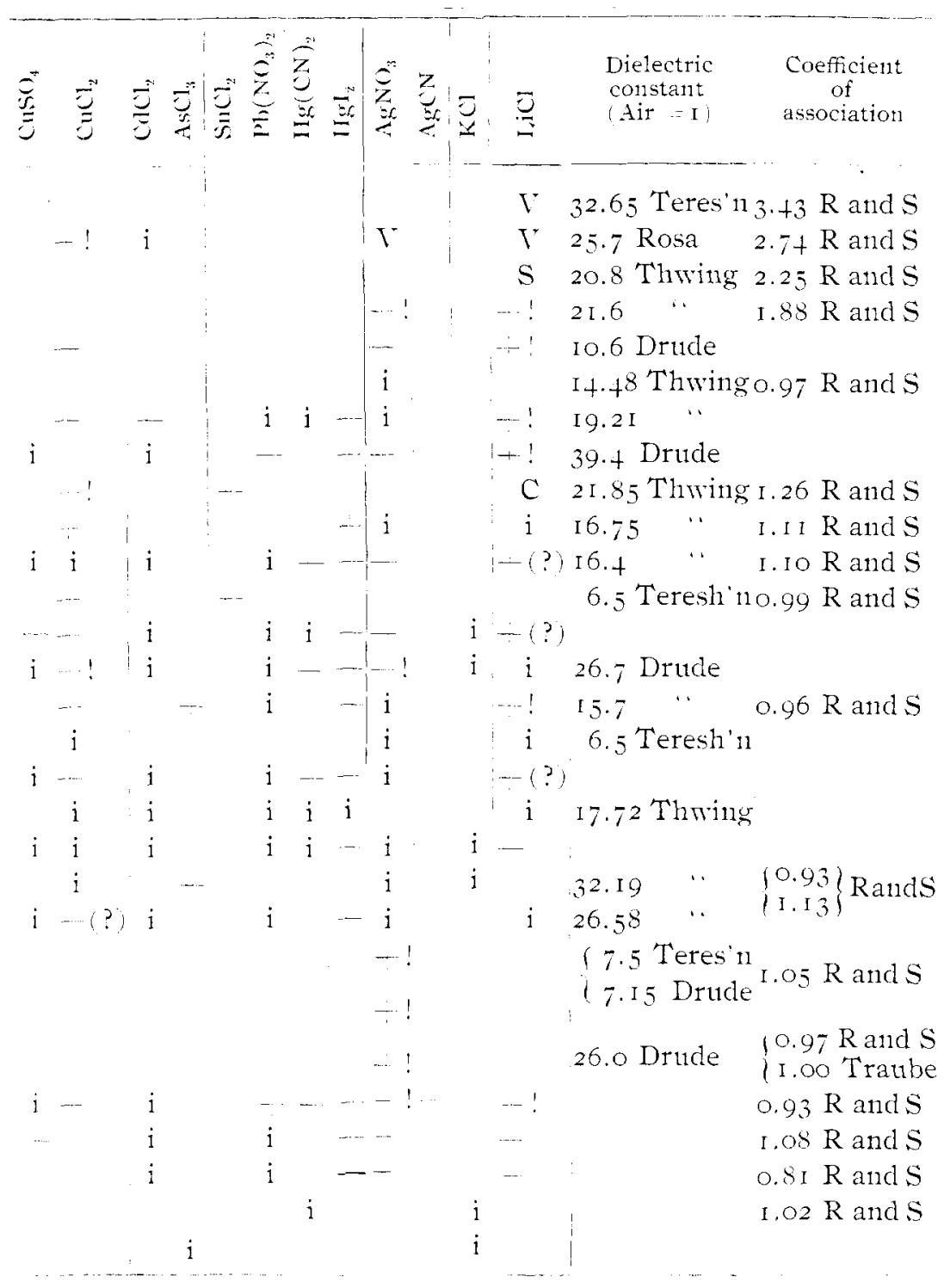


qualitative tests in this connection. Solutions of lithinm chloride in salicylic aldehyde conducts very well. The specific inductive capacity of furfurol would indicate that this solvent would rield solutions that conduct; and such is the case. The dissociative power of the solvents has been previonsly considered and consequently there are but few additions to be nuade to what has already been given, Silver nitrate is quite solnble in aniline and xylidine and both yield solutions that conduct very well. A large number of other tests were made which were too detailed. to be included in these tabulated results. Suffice it to say, however, that of the very large number of tests made both of organic and inorganic substances in these numerons solvents, there was not one instance when the solution conducted electricity, in which the dissolved substance was not an acid, a base, or a salt.

The additional results of the quantitative measurements of the electrical conductivity will now be given. A resistance cell of the Arrhenius pattern with the electrodes about three millimeters apart was used in making these determinations. The usual method of making the dilutions could not be employed owing to the small quantity of the solvents available. Into the resistance cell was introduced ten cc of the solvent by means of a carefully calibrated burette. The conductivity was measured and then a weighed portion of the salt was introduced, and when this was all in solution the conductirity was measured. Portions of the salt were successively introduced and the conductivity measurements made, until the requisite number of dilutions were made.

In the following tables $\approx$ represents the volume in liters in which one gram-nolecule was contained and $\mu$ the molecular conductivity expressed in reciprocal mercury units. The measurements were made at $25^{\circ}$ unless otherwise specified. The higher temperatures were obtained by heating the cell with its contents in a paraffin bath and the conductivity was measured as the temperature increased.

\section{ALLYL ALCOHOL}

A C. P. sample of allyl alcohol from Merck was treated with 
poatssium carbonate, over which it stood for several days. From this it was distilled and the distillate treated with fused potassimm hydroxide. The distillate from this was treated with canstic potash in contact with which it stood several days and it was then distilled. This distillate was redistilled twice and the conductivity determinations made as soon as possible. The specific conductivity was $6.5 \times 1 \mathrm{O}^{-5}$.

TABLE II

Solvent : allyl alcohol

\begin{tabular}{|c|c|c|c|}
\hline \multicolumn{2}{|c|}{ Ierric Chlorice, $\mathrm{FeCl}_{3}$} & \multicolumn{2}{|c|}{ Ferric Chloride, $(a)=20.02$ at $\left.25^{\circ}\right)$} \\
\hline 20.02 & $17 \cdot+2$ & $25^{\circ}$ & $17 \cdot+2$ \\
\hline $53.7 \mathrm{I}$ & 23.03 & 50 & $3+.62$ \\
\hline I 5.60 & 32.15 & 73 & +3.63 \\
\hline & & 80 & +5.65 \\
\hline
\end{tabular}

BENZYI, AICOHOL

The sample of benzyl alcohol employed was from Merck and was rectified by distillation. The portion used had a rery constant boiling-point. Oring to the diffeult solubility of the salts and the slight conductivity of solutions of this solvent, very few determinations were made. The specific conductivity was I. $76 \times 10^{-6}$.

\section{TABLE III}

Solvent : benzyi alcohol

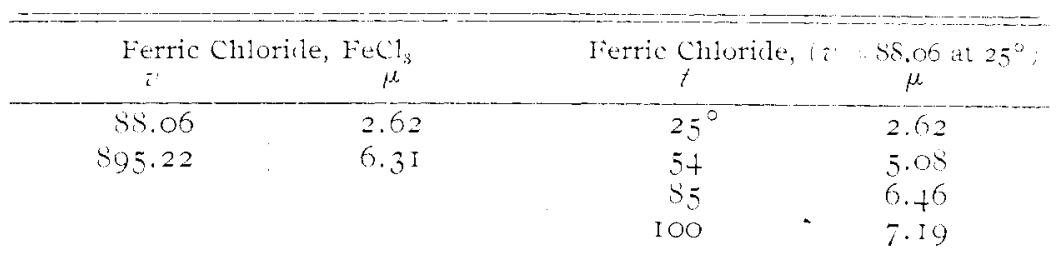

PARALIFHYDE

The sample of paraldelyde from Kahlbanm was distilled and the portion coming orer at a very constant temperature wan employed. The specific conductivity was less than 3.4 io $\mathrm{IO}^{- \text {- }}$ 
TABLE IV

Solvent: paraldehyde

\begin{tabular}{|c|c|c|c|c|c|}
\hline \multicolumn{2}{|c|}{$\begin{array}{l}\text { Ferric Chloricle, } \\
\mathrm{FeCl}_{3}\end{array}$} & \multicolumn{2}{|c|}{$\begin{array}{l}\text { Antimony Trichloride, } \\
\qquad \mathrm{SbCl}_{3}\end{array}$} & \multicolumn{2}{|c|}{$\begin{array}{c}\text { Antimony Trichloride } \\
\left(i^{\prime}=5.57 \text { at } 25^{\circ}\right)\end{array}$} \\
\hline & $\mu$ & $i^{\prime}$ & $\mu$ & $t$ & $\mu$ \\
\hline 4.37 & $9.8 \mathrm{I}$ & $5 \cdot 57$ & 0.202 & $25.2^{\circ}$ & 0.295 \\
\hline 21.32 & I $6.9 \mathrm{I}$ & 20.76 & 0.356 & 26.6 & 0.299 \\
\hline 42.52 & 18.76 & 61.16 & 0.532 & 29.0 & 0.298 \\
\hline 81.88 & 19. I 6 & & & 32.0 & 0.295 \\
\hline I 83.11 & I $6.5 \mathrm{I}$ & & & & \\
\hline 575.50 & I 6.9 I & & & & \\
\hline
\end{tabular}

The sample of salicylic aldehyde from Schuchardt was rectified by distillation. The portion taken had a constant boilingpoint and the specific conductivity was $5.98 \times \mathrm{IO}^{-6}$.

TABIE I*

Solvent : salicylic aldehyde

\begin{tabular}{cccc}
\multicolumn{2}{c}{ Ferric Chloride, ${ }^{2} \mathrm{FeCl}_{3}$} & Ferric Chloride, $\left(z_{t}^{\prime}=20.39\right.$ at $\left.25^{\circ}\right)$ \\
$v$ & 3.76 & $25^{\circ}$ & 3.76 \\
20.39 & $4.7 \mathrm{I}$ & 45 & 6.30 \\
81.38 & 5.60 & 50 & 6.73 \\
220.74 & & IO9 & 6.46
\end{tabular}

FL'RFLROL

The sample of furfurol from Merck was treated with fused calcium chloride, over which it stood for several weeks. It was then distilled and the distillate again distilled. The portion coming orer between $156^{\circ}$ and $15^{\circ}$ at $744 \mathrm{~mm}$ pressure was collected and its specific conductivity was $2.4 \times 10^{-5}$.

\section{TABLE VI}

Solvent: furfurol

\begin{tabular}{|c|c|c|c|c|c|}
\hline \multicolumn{2}{|c|}{$\begin{array}{l}\text { Ferric Chloride, } \\
\mathrm{FeCl}_{3}\end{array}$} & \multirow{2}{*}{\multicolumn{2}{|c|}{$\begin{array}{l}\text { Ferric Chioride, } \\
\left(a^{\prime}=45.60 \text { at } 25^{\circ}\right)\end{array}$}} & \multirow{2}{*}{\multicolumn{2}{|c|}{$\begin{array}{l}\text { Ferric Chloride, } \\
\left(z^{\prime}=45.60 \text { at } 25^{\circ}\right. \\
t\end{array}$}} \\
\hline$a^{\prime}$ & $\mu$ & & & & \\
\hline 45.60 & 20.78 & $25^{\circ}$ & 20.78 & $75^{\circ}$ & 47.96 \\
\hline 80.98 & 22.20 & 45 & 37.83 & 85 & $5 \mathrm{I} .4 \mathrm{I}$ \\
\hline \multirow[t]{2}{*}{$\mathrm{I} 49.2 \mathrm{I}$} & 26.42 & 55 & 40.98 & 95 & 56.07 \\
\hline & & 65 & 44.06 & 105 & 60.02 \\
\hline
\end{tabular}

1 Apparently this did not quite all dissolve. After cooling it became a riscons mass. 
ME'THYL-PROPYI, KETONE

The sample of methyl-propyl ketone employed was from Schuchardt and gave a specific conductivity of $9.5 \times \mathrm{IO}^{-7}$.

TABLE VII

Solvent: methyl-propyl ketone

\begin{tabular}{|c|c|c|c|c|c|c|c|}
\hline \multirow{2}{*}{\multicolumn{2}{|c|}{$\begin{array}{l}\text { Ferric Chloride, } \\
v^{\mathrm{FeC} 1_{3}} \mu\end{array}$}} & \multicolumn{2}{|c|}{$\begin{array}{c}\text { Ferric Chloride, } \\
\mathrm{FeCl}_{3}\end{array}$} & \multirow{2}{*}{\multicolumn{2}{|c|}{$\begin{array}{l}\text { Ferric Chloride, } \\
\left(v=13.64 \text { at } 25^{\circ}\right)\end{array}$}} & \multicolumn{2}{|c|}{$\begin{array}{l}\text { Ferric Chloricle } \\
\left(i^{\prime}=13.64 \text { at } 25^{\circ}\right.\end{array}$} \\
\hline & & $\ddot{i}^{\prime}$ & $\mu$ & & & $t$ & \\
\hline 13.64 & & & & $25^{\circ}$ & & $60^{\circ}$ & \\
\hline & & & & 49 & & 70 & \\
\hline & & & & & & & \\
\hline $100.7 \mathrm{I}$ & 42.76 & 1074.27 & 59.52 & 55 & 33.79 & 80 & 36.1 \\
\hline
\end{tabular}

$\mathrm{CuCl}_{2}$ in less tha11 460.28 liters gave $\lambda=5.22 \times$ IO $^{-6}$. A resistance of 6000 ohms was introduced in the measuring.

\section{ACETOPHENONE}

A sample of acetophenone from Schuchardt was treated with barium oxide, orer which it stood for several dars, and then distilled. The distillate was redistilled and the portion coming over between $194^{\circ}$ and $195^{\circ}$ at $745 \mathrm{~mm}$ pressure was taken. The specific conductivity of this portion was $1.8 \times 10^{-7}$.

$$
\text { TABLE VIII }
$$

Solvent : acetophenone

\begin{tabular}{|c|c|c|c|c|c|}
\hline \multicolumn{2}{|c|}{$\begin{array}{c}\text { Ferric Chloride, } \\
\mathrm{FeCl}_{3}\end{array}$} & \multicolumn{2}{|c|}{$\begin{array}{l}\text { Ferric Chloride, } \\
\left(i^{\prime}=23 .+6 \text { at } 25^{\circ}\right)\end{array}$} & \multicolumn{2}{|c|}{$\begin{array}{l}\text { Ferric Chloride, } \\
\left.\left(\sigma^{\prime}\right) 23.46 \text { at } 25^{\circ}\right)\end{array}$} \\
\hline$i^{\prime}$ & $\mu$ & $t$ & $\mu$ & $t$ & $\mu$ \\
\hline 23.46 & 10.28 & $25^{\circ}$ & 10.28 & $\mathrm{II}_{4}^{\circ}$ & $27 \cdot 49$ \\
\hline $46.7 \mathrm{I}$ & II.O3 & 50 & I 5.17 & I 25 & 28.74 \\
\hline 65.77 & I I. 59 & 65 & 17.52 & 1,35 & 28.85 \\
\hline $124.9 \mathrm{I}$ & 12.03 & 80 & 20.52 & 150 & 28.16 \\
\hline 292.98 & 13.08 & 100 & 23.90 & & \\
\hline
\end{tabular}

ETHYL MONOCHLORACE'TATE

This solvent was a C. P. sample from Schuchardt, the boiling-point of which was $143.5^{\circ}$. The specific conductivity was less than $1.7 \times 10^{-6}$. 
TABLE IX

Solvent : ethyl monochloracetate

\begin{tabular}{|c|c|c|c|c|c|c|}
\hline \multicolumn{2}{|c|}{$\begin{array}{l}\text { Ferric Chloride, } \\
\text { FeC1 }\end{array}$} & & \multicolumn{2}{|c|}{$\begin{array}{l}\text { Ferric Chloride, } \\
(a=7.76 \text { at } 25\end{array}$} & \multicolumn{2}{|c|}{$\begin{array}{l}\text { Antimony Trichloricle, } \\
\qquad \mathrm{SbCl}_{3}\end{array}$} \\
\hline 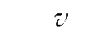 & $\mu$ & & $t$ & $\mu$ & $\theta$ & $\mu$ \\
\hline 7.76 & I 2.45 & & $25^{\circ}$ & $12 .+5$ & 4.25 & $0.17+$ \\
\hline $1+.96$ & $\mathrm{I}_{3} \mathrm{I} 4$ & & 50 & $\mathrm{I} 5.2 \mathrm{I}$ & I I. 49 & $0.20 \mathrm{I}$ \\
\hline 19.18 & I 3.49 & & 55 & 15.77 & $4+.73$ & 0.337 \\
\hline 22.09 & I 3.75 & & 67 & I 6.28 & \multirow{2}{*}{\multicolumn{2}{|c|}{$\begin{array}{c}\text { Cupric Chloricle. } \\
\mathrm{CuCl}_{\text {. }}\end{array}$}} \\
\hline+5.63 & $1+.78$ & & 80 & 16.28 & & \\
\hline 92.05 & 16.38 & & 92 & $\mathrm{I} 5.6 \mathrm{I}$ & " & $\mu$ \\
\hline \multirow[t]{2}{*}{ I 52.55} & 17.88 & & 100 & $1+.73$ & I $3 \cdot 32$ & I. 24 \\
\hline & & & I IO & $\mathrm{I} 3.1 \mathrm{I}$ & $!$ & \\
\hline
\end{tabular}

The $\mathrm{CnCl}_{2}$ did not quite all dissolve; so $\lambda=1.33 \times \mathrm{ro}^{-5}$.

\section{ETHYL CYANACETATE}

This solvent was a C. P. sample from Schuchardt and was rectified by redistillation. The boiling-point was very constant, and the portion collected came over between $203^{\circ}$ and $203.5^{\circ}$ at 74411111 pressure. The specific conductivity was $3.7 \times \mathrm{ro}^{-7}$.

\section{TABLE X}

Solvent : ethyl cyanacetate

\begin{tabular}{|c|c|c|c|c|c|c|c|}
\hline \multicolumn{2}{|c|}{$\begin{array}{c}\text { Silver Nitrate, } \\
\text { AgNO: }\end{array}$} & \multicolumn{2}{|c|}{ 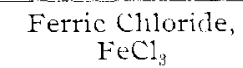 } & \multicolumn{2}{|c|}{$\begin{array}{l}\text { Ferric Chloricle, } \\
\left(\tau^{\prime}=15.30 \text { at } 25^{\circ}\right)\end{array}$} & \multicolumn{2}{|c|}{$\begin{array}{c}\text { Cupric Chloride, } \\
\mathrm{CuCl}_{2}\end{array}$} \\
\hline z & $\mu$ & $\bar{c}^{\prime}$ & $\mu$ & t & $\mu$ & $\tau^{\prime}$ & $\mu$ \\
\hline 10.62 & 4.19 & I 5.30 & 8.88 & $25^{\circ}$ & 8.88 & 29.85 & 7.00 \\
\hline 19.00 & 4.78 & 19.72 & 8.99 & +9 & I 3.40 & +1.67 & 7.50 \\
\hline 28.30 & 5.29 & 27.08 & 9.29 & 60 & 15.85 & $59 \cdot 40$ & 8.08 \\
\hline 58.57 & $6 .+6$ & 44.64 & 9.80 & 75 & 20.14 & 97.77 & I 2.80 \\
\hline \multirow[t]{4}{*}{ I 10.97} & 7.66 & 185.22 & I I .57 & 87 & $22 .+4$ & & \\
\hline & & & & 100 & $2+.83$ & & \\
\hline & & & & I 25 & $29 \cdot+9$ & & \\
\hline & & & & 150 & 38.70 & & \\
\hline
\end{tabular}

FTHYI OXAIATH

This solvent was a C. P. sample from Merck, and its specific conductivity was $7.12 \times 10^{-7}$. 


\section{TABLE XI}

Solvent: ethyl oxalate

\begin{tabular}{|c|c|c|c|c|c|c|c|}
\hline \multirow{2}{*}{\multicolumn{2}{|c|}{$\begin{array}{l}\text { Ferric Cliloride, } \\
\mathrm{FeCl}_{3}\end{array}$}} & \multicolumn{2}{|c|}{$\begin{array}{l}\text { Ferric Chloride, } \\
\mathrm{FeCl}_{3}\end{array}$} & \multirow{2}{*}{\multicolumn{2}{|c|}{$\begin{array}{l}\text { Ferric Chloricle, } \\
\left(a_{t}^{\prime} \text { I3 } 15 \text { at } 25^{\circ}\right)\end{array}$}} & \multicolumn{2}{|c|}{$\begin{array}{l}\text { Ferric Chloride, } \\
\quad \quad \text { I3 I5 at } 25^{\circ}\end{array}$} \\
\hline & & & $\mu$ & & & & \\
\hline I.3. I 5 & 5.88 & 94.79 & 6.25 & $25^{\circ}$ & 5.88 & $75^{\circ}$ & 10.15 \\
\hline 22.07 & 5.89 & $3+2.35$ & 7.70 & 50 & S.II & 100 & II.It \\
\hline+2.29 & 5.92 & & & 62 & $9.2 \mathrm{I}$ & 125 & 9.58 \\
\hline
\end{tabular}

(At $548^{\circ}$ solution began to boil.)

ETIYI, BEXZOATE

This sample was a C. P. sample from Trommsdorff, and its specific conductivity was about $1.8 \quad \mathrm{IO}^{-7}$.

\section{TABI.: XII}

Solvent : ethyl benzoate

\begin{tabular}{|c|c|c|c|}
\hline \multicolumn{2}{|c|}{$\begin{array}{c}\text { Ferric Chlorite, } \\
\text { Fec }\end{array}$} & \multicolumn{2}{|c|}{$\begin{array}{l}\text { Jerric Chloridle, } \\
\left(z^{\prime} \quad 29.5+\text { at } 25^{\circ}\right)\end{array}$} \\
\hline$i^{\prime}$ & $\mu$ & & $\mu$ \\
\hline $29.5+$ & I.5.5 & $25^{\circ}$ & 1.55 \\
\hline $17+28$ & $\mathrm{I} .6 \mathrm{I}$ & $5^{6}$ & I. I 6 \\
\hline 517.21 & I.9I & 75 & 0.82 \\
\hline & & 100 & $0 .+8$ \\
\hline
\end{tabular}

AMIL NITRITE:

This solvent was a C. P. sample from Schuchardt, and its specific conductivity was $1.8 \times \mathrm{IO}^{-7}$.

TABLE XIII

Solvent : amy' nitrite

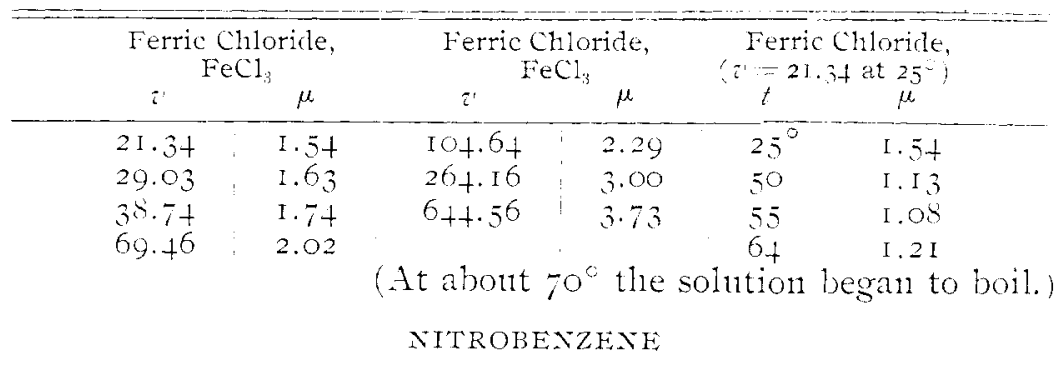

The sample of nitrobenzene employed was from Kahlbanm and was prepared from crystallizable benzene. The specific conductivity was less tha11 $3.5 \times$ IO $^{-i}$. 
TABLE XIV

Solvent : nitrobenzene

\begin{tabular}{|c|c|c|c|c|c|}
\hline \multicolumn{2}{|c|}{$\begin{array}{l}\text { Bismuth Trichloride, } \\
\mathrm{BiCl}_{2}\end{array}$} & \multicolumn{2}{|c|}{$\begin{array}{l}\text { Bismuth Trichloride, } \\
\mathrm{BiCl}_{3}\end{array}$} & \multicolumn{2}{|c|}{$\begin{array}{l}\text { Aluminium Chloride, } \\
\qquad \mathrm{AiCl}_{3}{ }^{\mathrm{T}}\end{array}$} \\
\hline$\tau^{\prime}$ & $\mu$ & i & $\mu$ & $\imath^{\prime}$ & $\mu$ \\
\hline 8.50 & 0.80 & 68.04 & I.03 & 4.69 & 3.67 \\
\hline $17.0 \mathrm{I}$ & 0.91 & I 36.07 & 1.07 & $9 \cdot 3^{8}$ & 4.51 \\
\hline $3+.02$ & 0.96 & 272.14 & I.I I & : & \\
\hline
\end{tabular}

ORTHO NITROTOLLENE

The C. P. sample of this solvent employed was from Kahlbaum, and the specific conductivity was less than $1.8 \times \mathrm{IO}^{-7}$.

\section{TABLE XV}

Solvent : ortho nitrotoluene

\begin{tabular}{|c|c|c|c|c|c|}
\hline \multicolumn{2}{|c|}{$\begin{array}{l}\text { Ferric Chlorile, } \\
\mathrm{FeCl}_{3}\end{array}$} & \multicolumn{2}{|c|}{$\begin{array}{l}\text { Ferric Chloride, } \\
\left(z^{\prime}=10.91 \text { at } 25^{\circ}\right)\end{array}$} & \multicolumn{2}{|c|}{$\begin{array}{l}\text { Antimony Trichloride, } \\
\mathrm{SbCl}_{3}\end{array}$} \\
\hline$v$ & $\mu$ & $t$ & $\mu$ & $\tau^{\prime}$ & $\mu$ \\
\hline 10.94 & 8.37 & $25^{\circ}$ & 9.39 & 3.40 & 0.056 \\
\hline 16.38 & 9.44 & 46 & I I. 98 & 6.55 & 0.088 \\
\hline 25.99 & 10.74 & 60 & 12.77 & 19.82 & 0.244 \\
\hline $7+.28$ & 13.32 & 70 & I 2.92 & 34.10 & 0.389 \\
\hline \multirow[t]{3}{*}{201.43} & 15.24 & 80 & I 2.67 & Mercuric & Chloride, $\mathrm{HgCl}_{2}$ \\
\hline & & 90 & I2. I 2 & | $\quad z$ & $\mu$ \\
\hline & & IOO & I 1.46 & 105.43 & 0.628 \\
\hline
\end{tabular}

META NTTROTOLLENE

The C. P. sample of this solvent was from Kahlbaum, and the specific conductivity was less than $\mathrm{I} .8 \times \mathrm{IO}^{-7}$.

\section{TABLE XVI}

Solvent: meta nitrotoluene

\begin{tabular}{|c|c|c|c|c|c|}
\hline \multicolumn{2}{|c|}{$\begin{array}{l}\text { Ferric Chloride, } \\
\mathrm{FeCl}_{3}\end{array}$} & \multicolumn{2}{|c|}{$\begin{array}{l}\text { Ferric Chloride, } \\
\left(2-10.86 \text { at } 25^{\circ}\right)\end{array}$} & \multicolumn{2}{|c|}{$\begin{array}{l}\text { Ferric Chloride, } \\
\left(\tau^{\prime}=10.86 \text { at } 25^{\circ}\right)\end{array}$} \\
\hline$i^{\prime}$ & $\mu$ & $t$ & $\mu$ & $t$ & $\mu$ \\
\hline IO 86 & 6.86 & $25^{\circ}$ & 6.86 & 80 & $\mathrm{r}_{5} . \overline{66}$ \\
\hline 46.93 & II.IO & 50 & I 2.28 & 90 & 17.35 \\
\hline 84.77 & I 2.55 & 60 & 13.05 & 100 & I 8.20 \\
\hline 448.14 & 19.00 & 70 & I 3.82 & I 25 & I 6.80 \\
\hline $8 \mathrm{I} 4.8 \mathrm{I}$ & I 8.20 & & & & \\
\hline
\end{tabular}

1 Aluminium chloride dissolved with evolution of heat. When the solution was diluted the evolution of hydrochloric acid gas was very perceptible, and for this reason no further determinations were made.

2 This solution remained in the resistance cell for two hours before the leterminations were made. 


\section{BFAZONI'TRIIE:}

The C. P. sample of this solvent emploved wasfrom Trommsdorff, and the specific conductivity was $1.9 \cdot 10^{-6}$.

\section{TABIF XYII}

\section{Solvent : benzonitrile}

\begin{tabular}{|c|c|c|c|c|c|c|c|}
\hline \multicolumn{2}{|c|}{$\begin{array}{c}\text { Silver Nitrate. } \\
\text { AgNO }\end{array}$} & \multicolumn{2}{|c|}{$\begin{array}{c}\text { Sirer Nitrate, } \\
\text { Ag }\end{array}$} & \multicolumn{2}{|c|}{$\begin{array}{l}\text { Silver Nitrate. } \\
\text { (a) } \quad 2.09 \text { at } 25^{\circ} \text {, }\end{array}$} & \multicolumn{2}{|c|}{$\begin{array}{r}\text { Silver Nitrate } \\
\therefore \quad 2.09: 2 \text { t } 25\end{array}$} \\
\hline$\because$ & $\mu$ & $i$ & $\mu$ & $t$ & $\mu$ & $t$ & $\mu$ \\
\hline 2.09 & 3.37 & 58.98 & II. I9 & $25^{\circ}$ & $5.55 ! ?$ & $95^{\circ}$ & 9.26 \\
\hline $9 .+3$ & 5.18 & 8.3 .92 & $5.3+1$ & 50 & 6.75 & 105 & 9.6 .5 \\
\hline 16.3 .3 & 6.37 & 151.96 & 16.38 & 56 & 7.05 & IIt & 10.09 \\
\hline 24.06 & 7.66 & 301.21 & I 8.20 & 65 & 7.59 & I 25 & 10.69 \\
\hline+4.62 & 10.12 & $50,3.24$ & 21.64 & 77 & 8.11 & 1,35 & I I. 20 \\
\hline & & & & 87 & $\therefore .75$ & 150 & I I. 70 \\
\hline
\end{tabular}

PYRIDINF,

The sample of prridine from König was fractionated, and the portion distilling over between Io $6^{\circ}$ and i i $7^{\circ}$ was treated with fused caustic potash from which it was distilled. The distillate was again treated with canstic potash, over which it stond for several days and was then distilled. The distillate was redistilled and the portion coning over between ir $3^{\circ}$ and I i $4^{\circ}$ at $7+2$ mm pressure was employed in some of the following determinations. The specific conductivity was 7.6 10-7. For other ceterminations a C. P. sample of the solvent from Kallban1m was employed, and its specific conductivity was about $7.5 \cdot \mathrm{IO}^{-}$.

TABIf XVIII

Solvent: pyridine

\begin{tabular}{|c|c|c|c|c|c|c|c|}
\hline \multicolumn{2}{|c|}{ leeric Chlorinte, } & $\therefore \quad \begin{array}{r}\text { Verric } \\
\lambda \text { of solvent }\end{array}$ & lerric Chlorile. & \multicolumn{2}{|c|}{ Ferric chlorite. } & \multicolumn{2}{|c|}{$\begin{array}{l}\text { Ferric } c h \text { horitle } \\
a^{\prime}=6.06 \text { at } 25^{\circ}\end{array}$} \\
\hline$i$ & $\mu$ & ') & $\mu$ & $t$ & $\mu$ & $t$ & $\mu$ \\
\hline \multicolumn{2}{|c|}{ i First Sieries } & \multicolumn{2}{|c|}{ Second Series) } & $25^{\circ}$ & 7.96 & $10^{\circ}$ & 21,16 \\
\hline 6.06 & 7.96 & +5.52 & 6.32 & $5+$ & 16.50 & $S 5$ & 22.71 \\
\hline $1.5,02$ & 7.52 & 93.69 & 5.91 & 50 & $17.2+$ & 90 & 23.36 \\
\hline $2+.56$ & 6.85 & 159.55 & 5.57 & 65 & 18.23 & 95 & 24,12 \\
\hline+2.18 & 6.82 & & & 70 & 19.59 & 100 & 24.80 \\
\hline \multirow[t]{2}{*}{95.35} & $6 .+1$ & & & 75 & 20.40 & 105 & 25.61 \\
\hline & & & & & & I IO & $25.6 \mathrm{I}$ \\
\hline
\end{tabular}




\begin{tabular}{|c|c|c|c|c|c|c|c|}
\hline \multirow{2}{*}{\multicolumn{2}{|c|}{$\begin{array}{l}\text { Silver Nitrate, } \\
\quad \mathrm{AgNO}_{3}\end{array}$}} & \multicolumn{2}{|c|}{$\begin{array}{c}\text { Silver Nitrate, } \\
\operatorname{Ag} N \mathrm{O}\end{array}$} & \multicolumn{2}{|c|}{$\begin{array}{c}\text { Lead Nitrate, } \\
\mathrm{Pb}\left(\mathrm{NO}_{3}\right)\end{array}$} & \multicolumn{2}{|c|}{$\begin{array}{l}\text { Mercuric Iodide, } \\
\mathrm{HgI}_{2}\end{array}$} \\
\hline & & $v$ & $\mu$ & $z^{\prime}$ & $\mu$ & $i^{\prime}$ & $\mu$ \\
\hline \multicolumn{2}{|c|}{ First Series } & \multicolumn{2}{|c|}{ Second Series } & 21.10 & $0.8 \mathrm{I}$ & $2 \mathrm{I} .78$ & 0.35 \\
\hline 7.55 & 24.07 & 392.28 & 40.16 & 55.96 & 1.57 & $93.6 \mathrm{I}$ & I. 39 \\
\hline $10.7 \mathrm{I}$ & 24.87 & 588.42 & +3.13 & I 68.47 & 3.25 & 200, I 2 & 2.70 \\
\hline 17.70 & 25.79 & 784.56 & 45.21 & \multirow{2}{*}{\multicolumn{2}{|c|}{$\begin{array}{l}\text { Cupric Chloride, } \\
\mathrm{CuCl}_{2}\end{array}$}} & \multirow{2}{*}{\multicolumn{2}{|c|}{$\begin{array}{l}\text { Silver Tartrate, } \\
\mathrm{C}_{6} \mathrm{H}_{4} \mathrm{O}_{6} . \mathrm{yg}_{3}\end{array}$}} \\
\hline $25 \cdot 52$ & 26.91 & \multirow{2}{*}{\multicolumn{2}{|c|}{$\begin{array}{c}\text { Silver Cyanide, } \\
A g C X\end{array}$}} & & & & \\
\hline $27 \cdot 43$ & 27.29 & & & $z^{\prime}$ & $\mu$ & & $\mu$ \\
\hline $37 \cdot 32$ & 27.96 & 2 & $\mu$ & 45.10 & 0.98 & 340.74 & I 4.64 \\
\hline $5 I .43$ & 29.49 & I 4.64 & 4.78 & 57.04 & I. I 6 & ${ }^{\mathrm{I}} 505.9 \mathrm{I}$ & 35.29 \\
\hline 60.90 & 30.17 & $2+\cdot 52$ & 5.04 & \multirow{2}{*}{\multicolumn{2}{|c|}{$\begin{array}{c}\text { Mercuric Cyanide, } \\
\mathrm{Hg}\left(\mathrm{CN}_{2}\right)_{2}\end{array}$}} & \multirow{2}{*}{\multicolumn{2}{|c|}{$\begin{array}{c}\text { Cobaltic Chloride, } \\
\mathrm{CoCl}_{2}\end{array}$}} \\
\hline $93.7 \mathrm{I}$ & 30.83 & 38.40 & $5 \cdot 4^{2}$ & & & & \\
\hline \multirow[t]{4}{*}{140.57} & 36.21 & 100.47 & 6.16 & $i^{\prime}$ & $\mu$ & i & $\mu$ \\
\hline & & 393.01 & 6.47 & $5 \cdot 35$ & 0.012 & 74.06 & 0.20 \\
\hline & & & & I 3.09 & 0.014 & $805.3 \mathrm{I}$ & I. 45 \\
\hline & & & & $357 \cdot 42$ & o. I 53 & & \\
\hline
\end{tabular}

A solution of cobaltic chloride becomes deep blue at a temperature between $40^{\circ}$ and $50^{\circ}$. The following specific conductivity determinations were made in order to ascertain whether the two differently colored solutions had the same conductivity.

Solvent

Saturated solution of $\mathrm{CoCl}_{2,}$ at $25^{\circ}$ $\lambda$ at $25^{\circ}$

\begin{tabular}{l|l}
$7.5 \times 10^{-7}$ & $1.4 \times 10^{-6}$ \\
$2.9 \times 10^{-6}$ & $5.5 \times 10^{-6}$
\end{tabular}

PIPERIDINE

A C. P. sample of this solvent from Kahlbaum was enployed, and its specific conductivity was less than $\mathrm{I} .8 \times \mathrm{IO}^{-7}$.

TABLE XIX

Solvent : piperidine

\begin{tabular}{|c|c|c|c|c|c|c|c|c|}
\hline \multicolumn{2}{|c|}{$\begin{array}{c}\text { Sil ver Nitrate, } \\
\mathrm{Ag}^{2} \mathrm{O}_{3} \\
\mu\end{array}$} & & \multicolumn{2}{|c|}{$\begin{array}{c}\text { Silver Nitrate, } \\
\mathrm{Ag} \mathrm{NO}_{3}\end{array}$} & \multicolumn{2}{|c|}{$\begin{array}{l}\text { Silver Nitrate, } \\
\left(a^{\prime}-4.24 \text { at } 25^{\circ}\right)\end{array}$} & \multicolumn{2}{|c|}{$\begin{array}{l}\text { Silver Nitrate, } \\
\left(a^{\prime}=4.24 \text { at } 25^{\circ}\right.\end{array}$} \\
\hline & & & $z^{\prime}$ & $\mu$ & $t$ & $\mu$ & $t$ & $\mu$ \\
\hline 4.24 & 0.368 & $\pi$ & 10.50 & $0.09 \mathrm{I}$ & $25^{\circ}$ & 0.368 & $40^{\circ}$ & 0.453 \\
\hline 5.25 & 0.277 & & I 5.62 & 0.043 & 30.5 & $0.39 \mathrm{I}$ & 45 & 0.478 \\
\hline 7.88 & O. I 54 & i & & & 35 & 0.432 & 50 & 0.508 \\
\hline & & & & $Q \mathrm{QI}$ & $\mathrm{INE}$ & & & \\
\hline
\end{tabular}

The sample of this solvent employed was from Merck, and its specific conductivity was $3.7 \times 1 \mathrm{IO}^{-7}$. 


\section{TABLE XX}

Solvent : quinoline

\begin{tabular}{|c|c|c|c|c|c|}
\hline \multicolumn{2}{|c|}{ 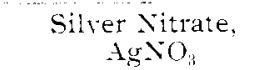 } & \multicolumn{2}{|c|}{$\begin{array}{l}\text { Silver Nitrate, } \\
\left(a^{\prime}=4.80 \text { at } 25^{\circ}\right)\end{array}$} & \multicolumn{2}{|c|}{$\begin{array}{l}\text { Silver Nitrate, } \\
\left(z^{\prime} \quad 4.80 \text { at } 25^{\circ}\right.\end{array}$} \\
\hline$i^{\prime}$ & $\mu$ & $t$ & $\mu$ & $t$ & $\mu$ \\
\hline+.80 & $2 .+5$ & $25^{\circ}$ & 2.45 & 85 & 5.89 \\
\hline 9.60 & 2.79 & 50 & 4.67 & 104 & 5.75 \\
\hline 34.92 & 2.80 & 62 & 5.20 & I $2 f$ & 5.77 \\
\hline I 29.83 & 3.62 & 70 & 5.52 & I 36 & 5.52 \\
\hline
\end{tabular}

Limiting values for the molectlar conductivity can be obtained in aqueous solutions, but this is, however, generally not the case in non-aqueous solutions. A few examples of those solrents that rield solutions that conduct the best will be given to illustrate this.

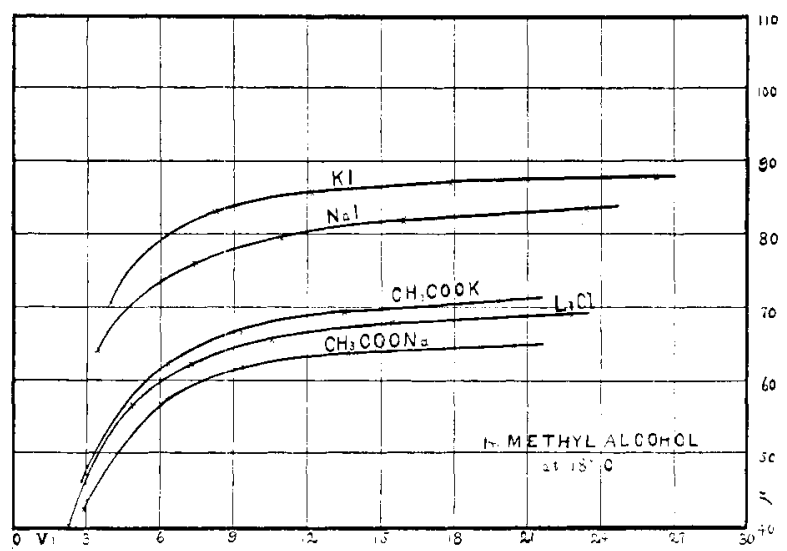

Fig. I

In Fig. I is plotted the molecular conductivity of various salts in methyl alcohol from the determinations of Vollmer at I $8^{\circ}$. The molecular conductivity is represented by the ordinates and the cube root of the volume in which one grammolecule of the substance was dissolved by the abscissas. The cube root of the rolume was employed in order to represent the values for the more dilute solutions in the figure. In Fig. 2 are plotted the results of Zelinsky and Krapiwin determined at $25^{\circ}$. In most cases, it will be observed that limiting values are 


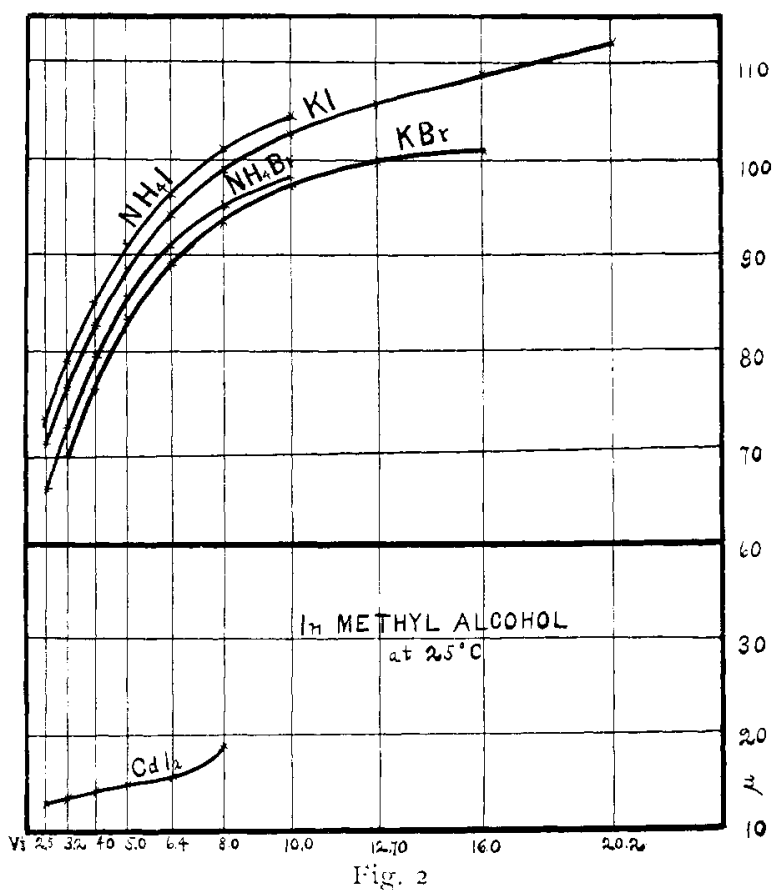

reached, $\mathrm{CdI}$, being, however, an exception, Zelinsky's and Krapiwin's determinations for KI indicate that the curve would probably not becone asymptotic and, therefore, no liniting value for $\mu$ wonld be reached. Nevertheless, Carrara assigns 97 as the ralne for $\mu_{x}$. Cohen has pointed out that the difference in the determinations of Zelinsky and Krapiwin and of Carrara may be attributed to the effects of the alcohol on the platinum black electrodes which they emplor. In Fig. 3 is plotted the molecular conductivity of various salts in ethyl alcoholic solutions from the determinations of Vollner at $8^{\circ}$. It will be noticed that the salts of the alkalies yield limiting values for $\mu$, while in the case of $\mathrm{CaCl}$ there seems to be no tendency for the curve to become asymptotic. The same is true for $\mathrm{Ca}(\mathrm{NO})$, and according to ny determinations for $\mathrm{FeCl}_{3}$ and $\mathrm{SbCl}_{3}$,

Carrara has calculated the values of $\mu$. for a number of halogen salts from Canpetti's deterninations of the transfer-

Nuovo Cimento [3] 35, 225. (Ref.) Jahr. ler Iilektrochemie, I, 22 (1894). 


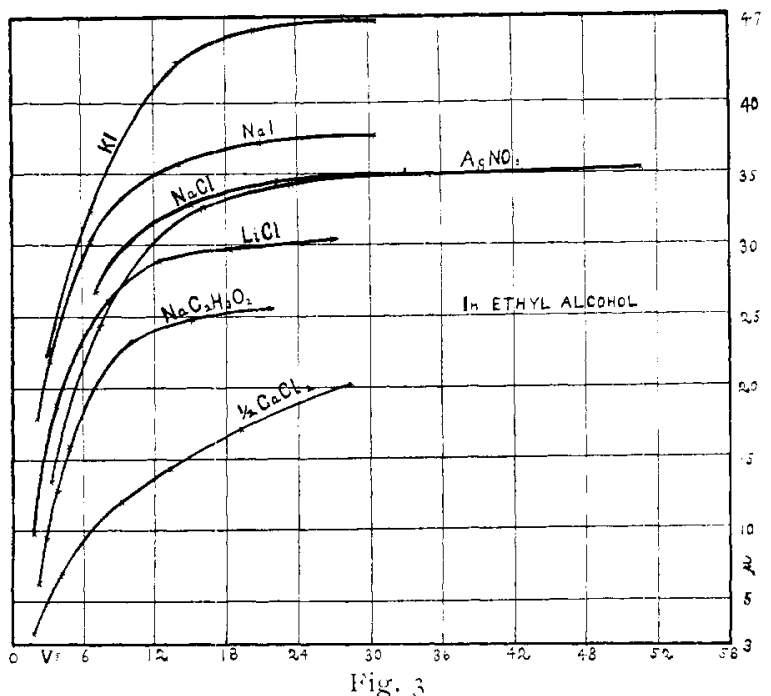

ence fignues of the hatogens in methylic alcohol solntions. $\mathrm{Ho}$ finds these values to agree very closely with his own experimental results. In the following table are given these results and also the value of $\mu$, in water.

\section{TABI.E: XXI}

Values of $\mu$.

In Ifethyl Alcohos

(I)

H

Li

Ia

K

$\mathrm{NH}$

$\mathrm{NCH}$

$\mathrm{N}\left(\mathrm{C}_{2} \mathrm{H}_{\mathrm{i}}\right)$

$\mathrm{S} / \mathrm{CH}_{5}$.

$\mathrm{H}$

$\mathrm{Li}$

$N a$

K

$\mathrm{NH}_{4}$

$\mathrm{N}\left(\mathrm{CH}_{3}\right)$

$\mathrm{N}: \mathrm{C}_{2} \mathrm{H}_{3}$

$\mathrm{S}(\mathrm{CH})$
$33 \times 0$

77.30

86.80

95.57

96.24

...

95.76

100.09

(1)

395.20

I 10.00

$119 .+0$

$1+0.80$

$1+0.6$

....

102.4

117.4
$\mathrm{Br}$

....

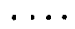

87.58

96.52

99.9 .3

- .

96.62

102.50

In Water

$\mathrm{Br}$

398.0

...

122.2

$1+3.6$

I +3.4

....

105.2

120.3
() H

$1.3+.50$

…

97.63

10.5 .25

II 5.30

I 13.76

I I 6.3

97.34
() I I

$\ldots$

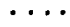

216.2

237.6

230.0

....

109.2

$2 \mathrm{I} 4 . \mathrm{O}$ 
From Campetti's results Carrara calculated the rate of migration of the ions in methyl alcoholic solutions and the results are given in the following table, which is taken from the Jahrbuch der Elektrochemie, 3, I3 (1896).

TABI, X XII

Speed of Migration

\begin{tabular}{|c|c|c|c|c|}
\hline & \multicolumn{3}{|c|}{ In Methyl Alcohol } & \multirow[t]{2}{*}{ In Water } \\
\hline & $\mathrm{Cl}$ & $\mathrm{Br}$ & I & \\
\hline $\mathrm{Li}$ & 27.83 & $\ldots$ & $\cdots$ & 39.8 \\
\hline $\mathrm{Na}$ & 37.33 & $37 \cdot 3.3$ & 37.33 & 49.2 \\
\hline $\mathrm{K}$ & 46.10 & 46.26 & 45.19 & 70.6 \\
\hline $\mathrm{NH}_{4}$ & 46.77 & +9.49 & $53.8 \mathrm{I}$ & 70.4 \\
\hline $\mathrm{N}\left(\mathrm{CH}_{3}\right)_{4}$ & $\ldots$ & $\ldots$ & 63.08 & +3.6 \\
\hline $\mathrm{N}\left(\mathrm{C}_{2} \mathrm{H}_{5}\right)_{4}$ & 46.29 & 46.36 & $6 \mathrm{I} \cdot 34$ & 32.2 \\
\hline $\mathrm{S}\left(\mathrm{CH}_{3}\right)_{3}$ & 51.43 & 49.85 & 63.94 & 47.6 \\
\hline $\mathrm{H}$ & 85.53 & $\ldots$ & $82.5^{\circ}$ & 325.0 \\
\hline $\mathrm{OH}$ & & 32.00 & & 170.0 \\
\hline $\mathrm{Cl}$ & & +9.47 & & 70.2 \\
\hline $\mathrm{Br}$ & & 50.24 & & 73.0 \\
\hline I & & $52 .+4$ & & 72.0 \\
\hline $\mathrm{CH}_{3} \mathrm{COO}$ & & 32.99 & & 38.4 \\
\hline $\mathrm{CCl}_{3} \mathrm{COO}$ & & 35.95 & & 32.8 \\
\hline
\end{tabular}

Kawalki found that the diffusion coefficient of a number of substances in ethyl alcohol is 0.34 times as great as in water. Vollmer from his work observed that the conductivity in ethyl alcoholic solutions at $18^{\circ}$ is approximately 0.34 times as great as in aqueous solutions, while his empirical factor for methyl alcohol is 0.73 . In general limiting values can be obtained for $\mu$ in methyl and ethyl alcoholic solutions except in the case of some salts of the heary metals.

In other alcoholic solutions no such uniformity seems to exist. This is perhaps best illustrated in the case of solutions in propyl alcohol. The molecular conductivity of solutions in this solvent is represented in Fig. 4. The determinations are from Schlamp's ${ }^{2}$ work. It will be noted that lithium salicylate is the only salt the conductivity of which appears to approach a

Wied. Ann. 52, 300 (1894).

Z Zeit. phys. Chem. 14, 272 (1894). 
limiting value. Schlamp does not hesitate, however, to assign values for $\mu_{i}$ in all cases, and from the plotted results this seems hardly justifiable. In the case of other alcoholic solutions, the

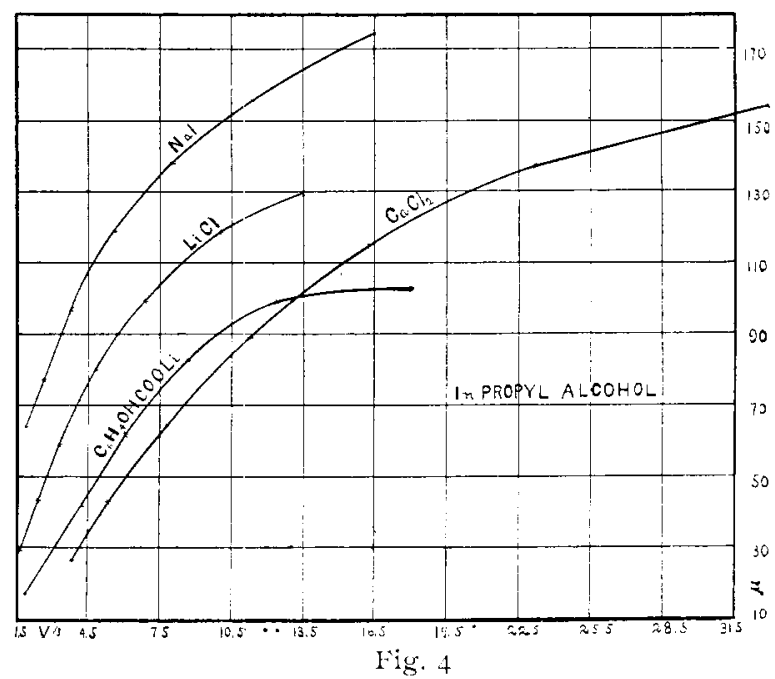

experimental eridence is so meagre that no generalization can be made (See Tables II, III).

The ketones were found to rield solutions that conduct electricity and this is in keeping with what the other investigators have found.

Acetone rields solutions the conductivity of which is, in general, better than in the case of many of the other hetones. In Fig. 5 is represented the conductivity of a number of salts in this solvent. The results plotted are from the determinations of Carrara principally; while those designated L are from Laszczynski, and those with D from Dutoit and Aston.

It will be noted that very few salts yield solutions in acetone that have limiting values for $\mu$. The values for two curves, for $\mathrm{NaI}$ and for $\mathrm{N}\left(\mathrm{C}_{2} \mathrm{H}_{5}\right)_{4} \mathrm{I}$, appear to indicate a decrease in the conductivity after certain dilutions are reached. The two curves

I ( ) Laszczynski. Zeit. Elektrochemie, 2, 55 (1S95). (2) Carrara. Gazz. Chim. Ital. 27, I, 207. (Ref.) Jahr. der Elektrochemie, 4, 48 (1897). (3) Du-

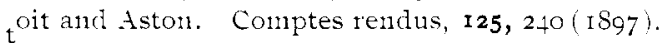


plotted for KI apparently intersect at about $\mu=153$. Laszczynski thinks the value of $\mu_{x}$ lies between i 60 and i 70 . Assunning the former value he calculates the factor $k$ in the formula

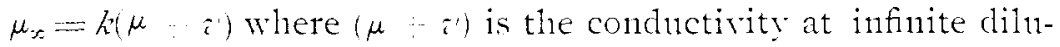
tion in water and $k$ is a constant. The value of $k$ he finds to be I.3. If this method be applied to salts other than the one he

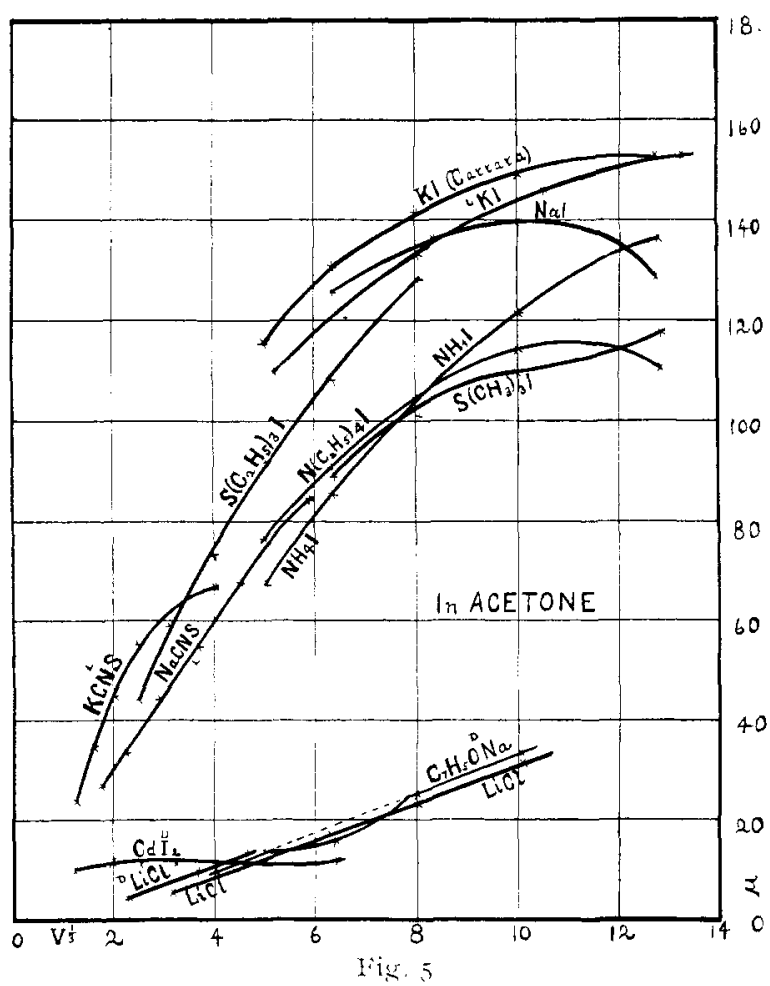

employed, $\mathrm{KI}$ - to CdI for instance, - it will be fonnd that different values for $k$ will be obtained. There is no such agreement between the limiting valnes for $\mu$ in acetone and in water as Vollmer found to hold in the case of ethy. alcohol and water.

In the other ketones the conductivity is not as great as in acetone, and while liniting values are obtained in sone cases, they are of the same order as CdI in acetone, that is, the value 
of $\mu$ remains virtually constant with the dilution. So in general limiting values in the ketones cannot be obtained.

Laszczynski and Corskir have measured the molecular conductivity of a number of salts in prridine and their results are plotted in Fig. 6. They assign liniting values to $\mu$ in the case

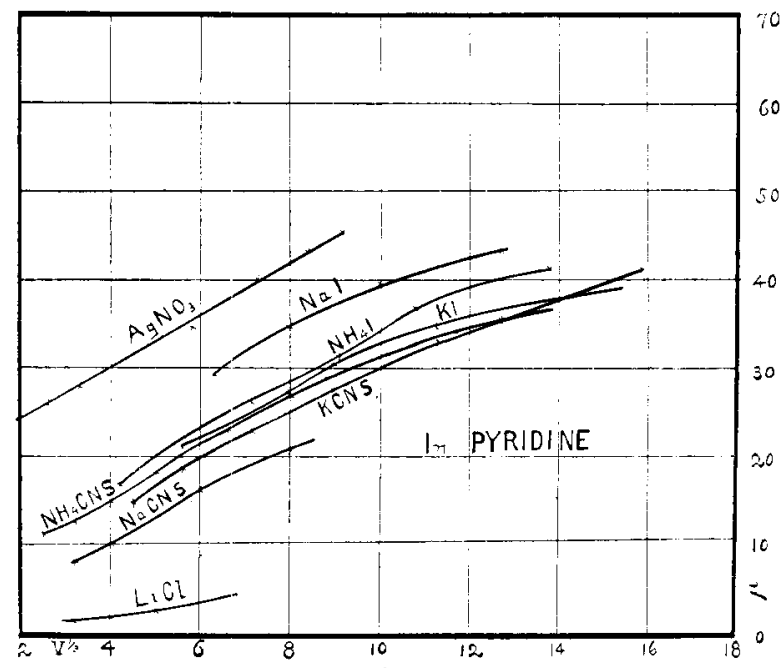

Iiig. 6

of $\mathrm{NH}_{4} \mathrm{CXS}, \mathrm{NH}_{4} \mathrm{I}, \mathrm{KI}$, and $\mathrm{NaI}$. It appears that the curves representing the conductivity of the solutions of these salts minght become asymptotic. The values for $\mathrm{AgNO}$ are $\mathrm{my}$ own determinations and these do not appear to approximate to a liniting value for $\mu$, at least not in the dilutions at which the measurements were made. The conductivity of the other salts in prridine is rather low and liniting values cannot, in general, be obtained.

Silver nitrate in benzonitrile does not yield limiting values for $\mu$. The solutions in the other nitriles conduct well, but they do not generally yield liniting values for $\mu$. Ferric chloride in nitrobenzene yields solutions in which the value of $\mu$ approaches a linit.

From the preceding it appears that there is no general n11i-

\footnotetext{
'Zeit. Elektrochenie, 4, 290 ( 1897 ).
} 
formity of limiting values in non-aqueous solutions as is found in the case of aqueous solutions.

The degree of dissociation in aqueons solutions is calculated from either the boiling-point or cryoscopic determinations or from the electrical conductivity of the solution. These methods yield concordant results, but in non-aqueous solutions this is not the case.

In order to calculate the degree of dissociation from the electrical conductivity measurements it is necessary to know the value of $\mu_{\%}$. It has been pointed out in the preceding that limiting values for $\mu$ are not always obtainable in non-aqueous solutions. In alcoholic solutions values for $\mu_{x}$ can generally be found. In the following table from Woelfer are given the values of the degree of dissociation, $a$, in methylic alcohol solutions as calculated from the boiling-point determinations of Woelfer, and from the conductivity measurements of Vollmer:

\section{TABLE XXIII}

$$
\text { Values of } a
$$

\begin{tabular}{lccc}
\multicolumn{1}{c}{ Salt } & Percent & $\begin{array}{c}\text { From } \\
\text { boiling-point }\end{array}$ & $\begin{array}{c}\text { From } \\
\text { conductivity }\end{array}$ \\
$\mathrm{LiCl}$ & 0.45 & 0.63 & 0.57 \\
$\mathrm{KI}$ & 0.36 & $0.6 \mathrm{I}$ & 0.79 \\
$\mathrm{NaI}$ & 0.44 & 0.87 & 0.74 \\
$\mathrm{CH}$ COOK & 0.48 & 0.48 & 0.63 \\
$\mathrm{CH}$ COONa & 0.40 & 0.49 & 0.63
\end{tabular}

The degrees of dissociation in methyl alcolnolic solutions as calculated from the boiling-point determinations, show closer agreement with those obtained from the conductivity determinations than in the case of the other alcoholic solntions.

Salvadorix found $\mathrm{HgCl}_{2}$ to be more highly dissociated in the methyl alcohol according to the boiling-point determinations than in aqueous solutions. Bechmann ${ }^{2}$ found the reverse to be true in ethyl alcoholic solutions. Jones and $\mathrm{K}^{\prime}$ ing ${ }^{3}$ calculated $712(1896)$

${ }^{1}$ Gazz. Chim. Ital. 26, I, 237 (I895). (Ref.) Jour. Chem. Soc. 7o, ii,

${ }^{2}$ Zeit. phys. Chem. 6, 437 (I890). 'An1. Chen1. Jour. I9, 753 (I897). 
the dissociation of $\mathrm{KI}$ in ethyl alcolool, from their boiling-point determinations, to be 25.4 percent in a 2 percent solution, and 27.2 percent in a 3 percent solution, $i$. e. the dissociation increases with the concentration. Cohen ${ }^{2}$ from the conductivity determinations at $\mathrm{I} 8^{\circ}$, found the degree of dissociation of $\mathrm{KI}$ to increase with the dilution, being abont 80 percent dissociated at a dilution of about rooo liters. This disagreement of the dissociation values obtained by these two methods will be made more apparent perhaps by Table XXIV, which is copied from Woelfer's paper. The results by the boiling-point method were obtained by himself, those by the conductivity method by Vollniner.

\begin{tabular}{|c|c|c|c|}
\hline \multicolumn{4}{|c|}{$\begin{array}{c}\text { TABLE XXIV } \\
\text { Values of } a\end{array}$} \\
\hline Salt & $\begin{array}{l}\text { l'ercent in } \\
\text { ethyl alcohol }\end{array}$ & $\begin{array}{c}\text { From } \\
\text { Doiling-point }\end{array}$ & $\begin{array}{c}\text { Froml } \\
\text { conductivity }\end{array}$ \\
\hline $\mathrm{LiCl}$ & 0.9 & 0.35 & 0.32 \\
\hline $\mathrm{CH}_{\mathrm{COOK}}$ & 1.07 & 0.18 & 0.27 \\
\hline $\mathrm{KI}$ & $0 .-8$ & 0.29 & $0 .+9$ \\
\hline $\mathrm{Ag} \mathrm{NO}_{3}$ & 0.53 & 0.65 & 0.38 \\
\hline $\mathrm{NaI}$ & $2 . \mathrm{I}+$ & 0.27 & 0.45 \\
\hline $\mathrm{NaI}$ & 0.68 & $0.5 \mathrm{I}$ & 0.56 \\
\hline $\mathrm{CH}_{3} \mathrm{COONa}$ & 0.97 & $0.0 \mathrm{I}$ & 0.24 \\
\hline
\end{tabular}

It is to be remembered that these two sets of values are calculated from results obtained at clifferent temperatures; in the case of the conductivity measurements at I $8^{\circ}$, when the viscosity factor is about $0.0 \mathrm{I} 2 \mathrm{II}$, and in the other case at $78^{\circ}$ when that factor had decreased (at $70^{\circ}$ to $0.0052 \mathrm{I}$ ). Yet it will be seen that there is no regularity of the results and that the degree of dissociation according to the boiling-point cleterninations is not invariably higher than that obtained by the conductivity method. It is no doubt but natural to ask, which of these methods gives the correct measure of the anlon11t of dissociation of the dissolved substances.

There have been too few molecules weight determinations of salts in the ketones made by the boiling-point method. This

1 loc, cit. 
method, according to Dutoit and Friderich ${ }^{\mathrm{r}}$, rields normal molecular weights for a number of salts when dissolved in acetone. These substances, with the exception of $\mathrm{LiCl}$ and $\mathrm{CdI}_{2}$, rield solutions that conduct fairly well. This wonld tend to indicate that they are quite highly dissociated while the boiling-point determinations indicate that they are not dissociated.

Attention has already been called to the fact of the conductivity of ferric chloride in nitrobenzene while the cryoscopic determinations indicate higher molecular weights than the theoretical.

In benzonitrile Werner ${ }^{2}$ found normal molecular weights for AgNO, while from Table XVII it will be seen that this solrent yields solutions of $\mathrm{Ag}_{\mathrm{N}} \mathrm{NO}_{3}$ that conduct well. It still remains to be seen what the molectlar condition of substances in other nitriles is according to the boiling-point determinations.

Wemer's boiling-point determinations show normal molecular weights for the salts of the heary metals in pyridine. The arerage of his determinations for $\mathrm{AgNO}$ is 165.4 , theory 169.55 ; for $\mathrm{Hg}\left(\mathrm{CN}, 2 \mathrm{I} 6.68\right.$, theory $25 \mathrm{r} .76$; for $\mathrm{HgI}_{2}, 308.0$, theory 452.88 ; and for $\mathrm{Pb}\left(\mathrm{NO}_{3}\right)_{2}, 352.07$, theory requires 330.35 . In most cases he obtained values a little nucler the theoretical. The greatest discrepancy accurs in the case of $\mathrm{HgCl}_{2}$, and it will be observed that the solution of this salt yields small values for $\mu$. From Table XVIII it will be observed that this solvent rields conducting solutions and since $\mu_{\%}$ is not known the degree of dissociation cannot be calculated.

From the preceding consideration it is apparent that there is not that agreenent between the degrees of dissociation as calculated from the boiling-point or cryoscopic determinations and from the conductivity measurements in non-aqueons solutions as has been found to hold in aqueous solutions.

In aqueons solutions the molecular conductivity always increase: with the dilution. From the analogy of the electrolytic dissociation of substances $i 1$ aqueous solutions to the dissociation

Bull. Soc. Chim. Paris 3) 19, 32 I i 1898 .

"Zeit anory. Chem. 15, I i 1897 ). 
of gases, Ostwald formulated a law of dilution for binary electrolytes which is as follows: $\mathrm{K}=-\frac{a^{2}}{(1-a) V^{2}}$; where $a: \mu_{\mu}^{\mu_{\text {r }}}$ and $V$ is the volume in which one gram-molectule of the dissolved stb. stance is contained. In aqueous solutions of weak electrolytes this law generally holds fairly well; and attempts have been made to apply it to non-aqueons solutions. Most investigators, Vollmer. Woelfer, Cattaneo, and others have found that Ostwald's dilution law does not hold for methyl and ethyl alcoholic solntions. Cohen has considered this subject at considerable length and connes to the sane conclusion.

Rudolphi's formula, which differs from Ostwald's in har.ing I V for $V$, has been shown to hold no better than Ostwald's. The values of the constant as calculated by these two formulat from the conductivity determinations of potassimm acetate are given in the following table which is taken from Cohen's article. $\mathrm{K}_{\mathrm{R}}$ indicates that those values were obtained by using $\mathrm{Ru}$ dolphi's formula while $\mathrm{K}_{\text {o }}$ indicates that Ostwald's was employed.

\section{TABI.E XXT.}

\begin{tabular}{|c|c|c|c|}
\hline$r$ & $\mu$ & $100 \mathrm{~K}_{12}$ & I00 K。 \\
\hline I I. & 8.28 & 0.82 & $0.2+2$ \\
\hline II 3.0 & 17.18 & 0.59 & 0.055 \\
\hline I I 20.0 & 27.00 & 0.49 & $0.01+$ \\
\hline $3,520.0$ & 29.20 & 0.36 & 0.006 \\
\hline
\end{tabular}

From the preceding it therefore appears that neither the dilution law of Ostwald nor that of Rudolphi holds for alcoholic solutions. It has been shown in the case of propyl alcoholic solutions that we are not justified in extrapolating values for $\mu_{r}$. hence the validity of the law of Ostwald and that of Rudolphi cannot be tested in resard to this solvent nor are the data sufficient to warrant conclusions being drawn concerning the applicability of these laws to other alcoholic solutions.

From Fig. IT it will be observed that the molecular conductivity increases with the dilution except in the case of CrI in acetone. The value of $\mu$ remains virtually constant and Dutoit and Friderich found the same to be true for solutions of 
this salt in acetophenone. In the case of solutions of $\mathrm{CdI}_{2}$ in methyl-propyl ketone and methyl-ethyl ketone the conductivity decreases with the dilution and the same was found by us to be the case for stannous chloride in acetone. Enler found the molecular conductivity of both $\mathrm{NaI}$ and $\mathrm{NaBr}$ in benzonitrile to decrease with the dihution. The conductivity of ferric chloride solutions in benzaldelyde decreases with the dilution and the same is true of solutions in pyridine. Silver nitrate in piperidine yields solutions the conductivity of which decreases with the dilution.

In face of these experimental results and in view of the fact that in non-aqueons solutions there are but few cases where values for $\mu_{*}$ can be obtained, it appears that neither the dilution law of Ostwald nor that of Rudolphi can hold in non-aqueons solutions.

In general the conductirity of non-aqueous solutions increases with the increase of the temperature. There are no exceptions to this in aqueous solutions, but in non-aqueous solutions there are a few. The molectlar conductivity of solutions of $\mathrm{CdI}_{2}$ in acetone remains the same at $50^{\circ}$ as at $25^{\circ}$; but it must also be remembered that the conductivity of this salt does not change with the dilution. Solutions of $\mathrm{CdI}_{2}$ in acetonitrile yield virtually the same values of $\mu$ at $0.2^{\circ}$ as at $37.2^{\circ}$. The values of $\mu$ for $\mathrm{SbCl}_{2}$ in paraldehyde are practically the same at $25.2^{\circ}$ as at $32^{\circ}$. In ethyl benzoate the conductivity of solutions of $\mathrm{FeCl}_{3}$ decreases from $\mathrm{I} .55$ at $25^{\circ}$ to 0.48 at $100^{\circ}$, and the conductivity of solutions of $\mathrm{FeCl}_{3}$ in anyl nitrite is practically constant at all temperatures tested.

There are not sufficient data to cletermine accurately the temperature coefficient of most of the non-aqueous solntions and particularly of the alcoholic solutions with which the most work has been done. But it can be stated that, in general, the conductivity does not increase proportionately with the temperature. It has been pointed out by Zelinsky and Krapiwin and later it has been confirmed by Cohen, as well as by Walker and Hann-

r Zeit. phys. Chem. 28, 619 (1899). 
bly, ${ }^{,}$that the temperature coefficient seems to be but slightly influenced by the addition of a non-electrolyte or even of a small quantity of water.

Although this investigation has been confined to the study of the conductivity of anhydrous salts and solvents it may be of interest to call attention to the work of some investigators on mixtures of water and alcohol. The following table of comparative conductivities is taken from the work of Zelinsky and Krapiwin. In the column headed $A$ is given the conductivity in aqueons solutions, under $B$ in methyl alcoholic solutions and under $C$ is given the conductivity in a $5^{\circ}$ percent solution of methyl alcohol and water.

TABLE XXII

\begin{tabular}{|c|c|c|c|c|c|c|c|}
\hline \multirow[b]{2}{*}{$\because$} & \multicolumn{3}{|c|}{$\mathrm{K} B \mathrm{r}$} & & \multicolumn{3}{|c|}{$\mathrm{XH}_{4} \mathrm{Br}$} \\
\hline & $A$ & $B$ & C & & $A$ & B & $c$ \\
\hline 16 & I $23 . \mathrm{I}$ & $\ldots$ & 59.82 & & 127.2 & 65.43 & 61.16 \\
\hline 32 & I 27.5 & 69.02 & 62.46 & & 131.8 & 72.73 & 63.81 \\
\hline 64 & I 30.5 & 76.70 & 65.36 & & 135.3 & 79.56 & 66.04 \\
\hline I 28 & 132.9 & 83.60 & 67 . I I & & 138.6 & 85.80 & 67.45 \\
\hline 256 & I 36.4 & 88.96 & 69.26 & & 141.2 & 90.88 & 68.32 \\
\hline $5 \mathrm{I} 2$ & I 40.2 & 93.26 & 70.53 & & 143.5 & 94.99 & 69.10 \\
\hline \multirow[t]{2}{*}{$102 t$} & $\mathrm{I}+3 .+$ & 97.25 & $\ldots$ & & 145.6 & 98.24 & $70 . \mathrm{II}$ \\
\hline & & $\mathrm{KI}$ & & & & $\mathrm{NH}_{k} \mathrm{I}$ & \\
\hline$i^{\prime}$ & $A$ & $B$ & C & & $A$ & $\mathrm{~B}$ & c \\
\hline 16 & 124.5 & 69.20 & 62.13 & & I 25.4 & 72.24 & 62.63 \\
\hline 32 & I 28.2 & 76.35 & 64.3 & & 129.6 & 78.74 & 65.04 \\
\hline 64 & 130.5 & 82.52 & 66.01 & & I 33.4 & 85.0 & 67.48 \\
\hline I 28 & 133.0 & 88.69 & $67 .+5$ & & 135.9 & $9 \mathrm{II} 4$ & 69.28 \\
\hline 256 & 135.8 & 93.85 & 68.28 & & 138.7 & 96.20 & 70.34 \\
\hline 512 & 137.9 & 98.19 & 69.65 & & $14 \mathrm{I} .3$ & 100.6 & $7 \mathrm{I} . \mathrm{I} 2$ \\
\hline IO24 & 140.9 & 102.2 & 70.55 & & $1+3.7$ & 104.7 & 71.57 \\
\hline
\end{tabular}

It will be noticed that the conductirity of the halogen salts of the alkalies in methyl alcohol (B) is considerably less than in aqueons solutions (A). When water is added to the extent of $5^{\circ}$ percent even (C), the molecular conductirity is somewhat less than it is in methyl alcohol. Colen and others have pointed out the same fact; that is, at i $8^{\circ}$ the conductivity of a mixture of water and alcolnol, containing more than 60 percent of alcohol,

\footnotetext{
'Jour. Chem. Soc, 71, i, 66 (1897).
} 
is less in dilute solution than in absolute alcohol. This fact seems to be rather difficult to reconcile with the electrolytic dissociation theory, for here we have two solvents that possess dissociative power in a high degree, and yet a salt dissolved in a mixture of equal parts of these rields a solution the conductivity of which is less than that of the solutions formed when dissolved in either.

Carrara has shown that the electrolytic dissociation of water in methy alcohol is greater than in aqueous solutions, while the reverse is the case in ethyl alcohol. It is also of interest to note that $\mathrm{KOH}$ and $\mathrm{NaOH}$ in methyl alcohol show the same conductirity as $\mathrm{CH}_{3} \mathrm{OK}$ and $\mathrm{CH}_{3} \mathrm{ONa}$.

As has been previonsly noted, ${ }^{2}$ Nernst calls attention to the fact that solvents which have a high specific inductive capacity, rield solutions that conduct; and he argues that the greater the dielectric constant of a solvent, the greater is the power of dissociation. In general, those solvents that have a high dielectric constant do yield solutions that conduct, but the molecular conductivity is not commensurate with the dielectric constant. Lithium chloride in an aqueous solution (dielectric constant of $\mathrm{H}_{2} \mathrm{O}$ is 78 ), gives a value of 95 for $\mu_{x}$ at I $8^{\circ}$; while in propyl alcohol (dielectric constant is 20.45 ) in a volume of about 3000 liters, the value of $\mu$ at $I 5^{\circ}$ is given as I 28.9. In fact, nost of the values of $u$ in dilute solutions of propyl aicohol are, according to Schlamp, greater than the values of $\mu$, in water. In methyl alcohol (dielectric constant is 32.65 ) the value for $\mu_{\text {s }}$ for $\mathrm{N}\left(\mathrm{C}_{2} \mathrm{H}_{5}\right)_{4} \mathrm{I}$ is I I 3.8 , while in water it is 107.6; for $\mathrm{N}\left(\mathrm{CH}_{3}\right)_{4}$ I the values of $\mu_{\infty}$ in both solvents are virtually the same (I $x_{5} .3$ ). In other alcoholic solutions the values of $\mu$ do not even approximate to those in aqueous solutions, however.

Esters, the dielectric constants of which are very low, yield solutions that conduct, - for example - ethyl oxalate, ethyl benzoate, and ethyl acetate. Attention has previously been called;

${ }^{1}$ Gazz. Chinn. Ital. 27, I, 422 ( I 897 ). (Ref.) Jour. Chenı. Soc. 72, ii, 473 (1897)

: Jour. Phys, Chem. 3, I2 ( I 899 !.

"Ibid. 3, 2I ( I 899 ). 
to the fact that ethyl acetoacetate yields solutions of ferric chloride that conduct better than those in ethyl cyanacetate, the dielectric constant of the latter being 26.7 , while that of the former is only 15.7 . The values of $\mu$ in acetone approximate to and in some cases exceed the values in methyl alcolnolic solutions. Benzonitrile was the only nitrile the dielectric constant of which could be found in the literature, but it is probable that the value of the other constants is relatively high. The value of $\mu$ for acetonitrile solutions approximate closely to those obtained for aqueous solutions, while for $\mathrm{CNS}_{\mathrm{NHH}}$ and $\mathrm{NaI}, \mu$ is of about the same magnitude as for $\mathrm{AgNO}_{3}$ in benzonitrile. The dielectric constants of prtidine, piperidine and of quinoline conld not be found in the literature.

From these considerations it therefore appears that while those solvents which have a high specific inductive capacity are the ones which, in general, yield solutions that conduct the best, the magnitude of $\mu$ is not proportional to the value of the dielectric constants.

Dutoit and Fiderich, ${ }^{1}$ from the results of other investigators and from their own researches on the ketones and nitriles, conclude that "la conductibilité des electrolytes dissons dans un dissoliant non poly'mérisé est mull." The degree of accuracy of this statement will become manifest when the following table is examined. In Table XXVII are arranged the names of a number of solvents in the order of increasing coefficients of association. The names appear in the first columm, the coeffcients of association in the second. These values were mostly. cletermined by Rannsay and Shields (See Table I). The third column contains the viscosity factors, $\eta$, as far as they conla be obtained from the tables of Dutoit and Friderich and of Iandolt and Börnstein. The last three columns contain the formula of the salts, the volume in liters in which one gram-molecule was dissolved, and the molecular conductivity at that particular concentration. That salt was nstrally chosen which gave the largest value for $\mu$.

\footnotetext{
' Buil Soc. Chim. Paris, 3 I9. 321, (18gs).
} 


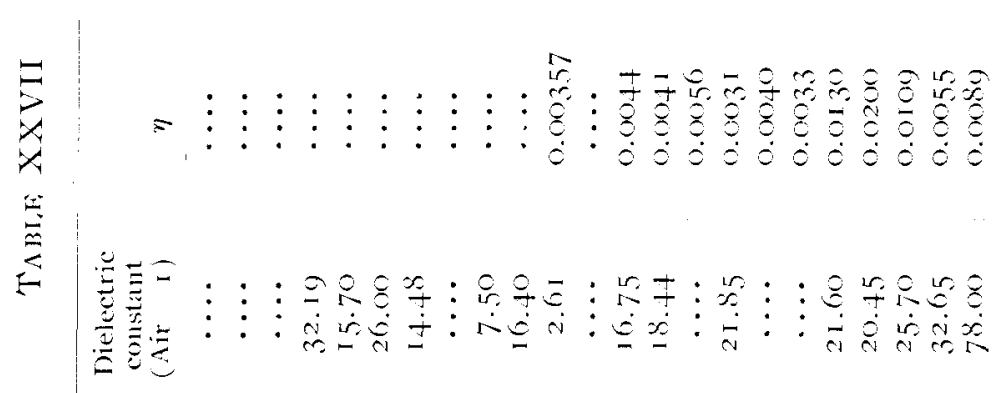

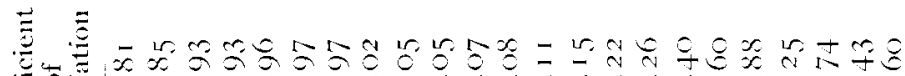

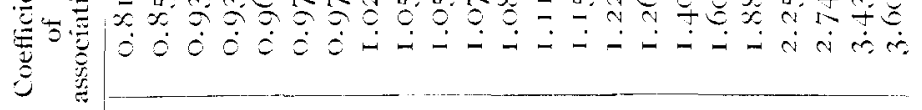

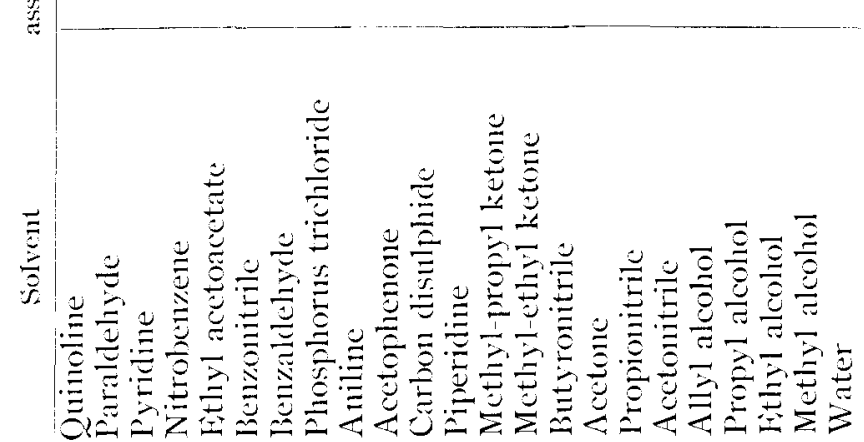


It will be observed that those solvents down to and including benzaldehyde, are not polymerized liquids according to the values of their coefficients of association, and they all produce solutions that conduct electricity. Among these are benzaldehyde and paraldehyde, both of which yield solutions that conduct well. Benzonitrile, the coefficient of association of which, even according to Traube, shows virtually no polymerization, rields solutions that conduct very well; and according to Werner gives normal molecular weights by the boiling-point method. Further, ethyl acetoacetate yields solutions that conduct very well; yet its molecules are not polymerized. It will also be noted that all of these solvents have relatively high dielectric constants. Solutions in nitrobenzene conduct; but according to Ramsey and Shields its molecules are not polymerized. Traube, however, gives a value of 1.47 for the coefficient of association of this substance. Of the organic bases quinoline rields solutions that conduct and shows the lowest degree of association of any of the solvents. Pyridine dissolves a large number of salts, and yields solutions that conduct; ret its molecules are not polymerized. It wiil be seen that the group of solvents whose coefficients of association are between I and I.OS inclusive, thus indicating a very slight degree of polymerization, rield solutions that conduct slightly, and Ramsey and Shields regard most of these as non-polymerized liquids. Aniline yields solutions that conduct, particularly solutions of silver nitrate; acetophenone solutions do not conduct very well; and those in piperidine conduct poorly; while phosphorus trichloride and carbon disulphide, whose molecules are slightly polymerized, do not yield solutions that conduct electricity.

The molecular conductivity of solutions of different solvents is not commensurate with the degree of polymerization of their molecules. The examples given to illustrate that the molecular conductivity is not proportional to the dielectric constants of the solvents are applicable here as well. The value of $\mu$ depends, however, to a great extent upon the salts chosen; for example, the molecular conductivity of $\mathrm{CdI}_{2}$ is virtually constant (I I.7) in 
acetone and in propionitrile it is 19.2 at dilution 5 I 2 liters; while the molecular conductivity of silver nitrate is 35 in ethyl alcohol at dilution 4I,000 liters and I 59 in acetonitrile at I, I 4 I liters. The coefficient of association of ethyl alcohol is 2.74 and that of acetonitrile is r.60.

The coefficients of association as determined by Ramser and Shields hare been employed in preference to those by 'Traube' becalse the method of the former has a better theoretical basis, and their results are more in accord with those of other investigators. Traube gives for the value of the coefficient of association for benzene I.I8, for toluene I.o8, for ethylene chloride I.46; and not any of these solvents yield solutions that conduct electricity. Then he gives values for the esters that are very much in excess of the determinations of other investigators, whereas the values for the alcohols are very much less. For example, for methyl alcohol he gives as the coefficient of association I.79, for ethyl alcohol 1.67, for propyl alcohol 1.55 , and for water 3.06. (Compare with the values given in Table I.)

IIany solvents, whose molecules are polymerized, yield solutions that conduct electricity, and there are some whose molecules are supposed to be polymerized that do not yield solntions that conduct: and if Traube's results be taken, a large addition to those cited above could be given. It has also been pointed ont that there are a considerable number of solvents, whose molecules are apparently not polymerized, yet whose solutions conduct electricity well,-for example, benzonitrile, ethy] acetoacetate, etc.

From the considerations presented in the preceding it appears that the theory as promnlgated by Dutoit and Friderich, that only polymerized solvents yield solntions that conduct, is untenable.

Sometime before Dutoit and Fricierich published their conclusions, Crompton1 ${ }^{2}$ assigned a wider rôle to the associative property of liquids. He presents the riew, that by means of this

${ }^{1}$ Ber. chem. Ges. Berlin, 30, 273-4 is 897 !.

${ }^{2}$ Jour. Chenn. Soc. 7 I, ii, 925 I 897 I. 
theory of association the anomalous results obtained in the case of certain dissolred substances, electrolytes, by the boiling-point. freezing-point and osmotic pressure determinations, can be easily. explained; and aims to show that the electrolytic dissociation theory is not only unnecessary but in many respects inadequate. As has been previonsly pointed ont no proportionality exists between the values of the dielectric constants of the solvents and the molecular conductirity of their solutions. Crompton calls attention to the connection between the specific inductive capacity and the degree of association of the solvents. This parallelism has also been pointed out by Abegg.s who further observes that nitrobenzene, ethyl nitrite and benzonitrile all have high dielectric constants; yet their association factor is unity. This, he thinks, fulfills the primary conditions of a self-dissociating substance. Crompton further states: "It is almost impossible to doubt that association plays an all-important part in determining the value of the specific inductive capacity of a liquid, and that if there is any connection between the specific inductive capacity and the power of forming electrolytes, it may be looked for rather in the fact that electrolytes are solutions of approximately monomolecular salts in an associated solvent, than in there being any peculiar 'dissociative power' attached to the solvent." From the experimental results herein presented it appears that it is a fact, that all solvents that yeld solutions which conduct are not associated liquids.

Crompton further aims to explain the conductivity of fused salts upon the dissociation of the associated molecules of the fused salts, wherein a small proportion of the salt is in the monomolecular condition. Abegg, howerer, shows that in many. cases the melted salt has a higher dielectric constant than its "ice,"-for example, the dielectric constant of water is 78 while that of ice is 2.85 according to Thwing; and that in these fused salts the self-ionization or self-dissociation is very slight. He states that in about Ioo liters of fused $\mathrm{AgCl}$ there is abont one gram-molecule of $\mathrm{AgCl}$ completely dissociated. Kohlransch"

\footnotetext{
' Zeit. Electrochemie, 5, 48 (1899).

"Wiect. Ann. 53, 209 i I894!.
} 
has shown that in about eleven million liters of water there is one gram of hydrogen ions; while in methyl alcohol Carrara ${ }^{2}$ found one gram-molecule of methyl alcohol dissociated in about five and a half million liters. If water and methyl alcohol manifest such great dissociative power upon salts dissolved in them, why is it that they do not dissociate their own molecules to a greater extent?

It is quite noticeable, that a large number of the investigators of the properties of non-aqueous solutions, express the thought that there is manifested considerable influence between the dissolved substance and the solvent. This factor of the influence of the solvent upon the dissolved substance, is one that is no doubt of very great importance; and in the development of the electrolytic dissociation theory (which is based upon the behavior of aqueous solutions) the action of the solvent upon the dissolved substance has been entirely neglected. The opponents of the dissociation theory present this fact in rather forcible manner. ${ }^{2}$

Fitzpatrick ${ }^{3}$ concludes from his investigation on the conductivity of alcoholic solutions that the action of the solvent upon the dissolved substance is a chemical one. He conceives the dissolved salt as decomposing and forming molecular groups in the solvent. Owing to the large excess of the solvent there will be a continual decomposition and recombination of these molecular groups. He cauticns one against regarding the solvent as a medium in which the salt particles are suspended or as a dissociating agent. Wildermann, ${ }^{4}$ on the other hand, recognizes two kinds of dissociation-one, the electrolytic dissociation of the dissolved substance, and the other, the dissociation of the iarger molecular aggregates into smaller ones. For example, in a solution of $\mathrm{KCl}$ in water the following aggregates are assumed to exist: $\mathrm{K}_{2} \mathrm{Cl}_{2}, \mathrm{KCl}, \mathrm{K}_{2} \mathrm{Cl}, \mathrm{KCl}_{2}, \overline{\mathrm{K}}$ and $\overline{\mathrm{C}}$. $\mathrm{He}$

${ }^{1}$ Gazz. Chin. Ita1. 27, I, 422 (1897). (Ref.) Jonr. Chem. Soc. 72, ii, 473 (1897).

"Fitzgerald's "Helmholtz' Memorial Lecture" Jour. Chem. Soc. 69, i, 885 (1896).

1oc. cit.

'Ber. chem. Ges. Berlin, 26, r773, 288 I (I 993 ). 
further maintains that solutions of all substances, whaterer the solvent or the concentration, undergo electrolytic dissociation.

Cattaneo $^{I}$ was impressed with the fact that the molecular conductivity is greatly influenced by the nature of the solvent employed. He was not able, however, to point ont any direct relation existing between the varions properties of the solvents which yield solutions that conduct electricity. Konovaloff ${ }^{2}$ from his work on the amines, concludes that only those solvents that react chemically with the dissolved substances rield solutions that conduct. It is true that there are many solvents of this nature which do react with the dissolved substance, and ret which do not yield solutions that conduct electricity. Picric acid reacts with benzene but the resulting solution does not conduct. Hence chemical combination of the dissolved substance with the solvent may take place and yet the solutions need not necessarily conduct. Werner ${ }^{3}$ has isolated and analyzed a large number of products of pyridine and piperidine anlong other organic solvents, with salts of the heary metals. From the boiling-point determinations, the molecular weights of these salts seem to be very slightly influenced by their union with the solrent. This is analogons to the fact, that salts which crrstallize from an aqueous solution with water of crystallization, yield the same molecular weight whether dissolved in the anlydrous form or with their water of crystallization. Carrara thinks that the union of solvent and dissolved substance accounts for the slight conductivity in certain cases. The low values of $\mu$ in the case of acetone solutions of hydrochloric acid and lithium chloricle he attributes to this fact.

It has been pointed ont by Cianiciant that the dissociative power of a solvent depends principally upon its chemical structure. That is, componnds of the same chemical type, for exanple, of the $\mathrm{HOH}$ type, yield solutions that conduct electricity

' Rench. Accad. I,inc, (5) 4, II, 63, 73 (I\$95) (Ref, ) Jour. Chem. Soc, 72, ii. 537 (I 897 ).

"Wierl. An11. 49, 733 (1893).

"1oc. cit.

'Zeit. phys. Chem1, 6, 403 (1S9o:. 
well. This is true in the case of alcoholic solutions, which are not the only class of conpounds, however, that possess dissociative power, as has already been pointed ont. In general, however, if one member of a particular type of componnds (e. g. nitriles) yield solutions that conduct, it has been found that other members also possess this property; and if a member of some other type (e. g. hydrocarbons) is found not to yield solntions that conduct, other members do not possess dissociative power.

The data collected are as yet insufficient to show what the relation between solvent and dissolved substance nutst be in order to yield solutions that conduct electricity. Enough facts have been presented, however, to make it apparent that any theory that aims to explain the electrical conductivity of solutions in general, must take into consideration the influence of the solvent upon the dissolved substance. This subject is replete with interest, for closely connected with it is the true cause of the solubility of substances.

From the foregoing the following general statements may be made:

I. In methyl and ethyl aicoholic solutions liniting values for $\mu$ can usually be obtained. The salts of the heavy metals are apparently exceptions. In other solvents a liniting value is very seldom obtained.

2. The degrees of dissociation of the dissolved substances in non-aqueons solutions, as calculated from the boiling-point or cryoscopic determinations and from the conductivity measurements, show very great disagreement. No such agreenent is manifest as is observed in the case of aqueons solutions.

3. Neither the dilution law of Ostwald nor that of Rudolphi lolds in the case of alcoholic solutions. In other solvents (since the conductivity remains virtually constant in some cases with the increased dilution, while in others it decreases) it is very apparent that the above-named laws do not hold. Then, too, since the value of $\mu_{x}$ cannot be obtained in the case of so many solvents, the validity of the dilntion laws conld not be tested. 
4. The molecular conductivity, in general, increases with the dilution; but in several cases the conductirity decreases with the successive dilutions.

5. The molecular conductivity usually increases with the temperature, but not proportionally. In some cases it remains virtually constant with increase of temperature, while in others it decreases. The changes in the riscosity accompanying a varying temperature have not been determined.

6. Solvents that have a high dielectric constant yield solutions that conduct the best. Some solvents, the dielectric constants of which are very low, give poorly conducting solutions. The molecular conductivity is not, however, proportional to the dielectric constant of the solvent.

7. Some associated solvents rield solutions that conduct electricity, whereas others do not. Some solvents whose molecules are not polymerized yield conducting solutions. The value of $\mu$, in those solutions that conduct, is not commensurate with the degree of association of the solvent.

8. The conductivity of electrolytes depends very much upon the nature of the solvent employed. No rigid classification can be made, but, in general, solutions in the hydrocarbons and their halogen substitution-products do not conduct. Alcoholic solutions conduct well, and the conductivity, in general, decreases with the increase of the carbon content. Solutions in esters conduct, but those of high carbon content usually vield solutions that conduct very poorly. The ketones and the aldehydes yield solutions that conduct well. Of the nitrogen compounds, liquid ammonia and ammonia substitution-products, or anines, conduct. The nitriles of both the aliphatic and aromatic series yield conducting solutions; and of the organic bases pyridine and quinoline come under this same category.

9. The dissociation theory as promulgated for the explanation of the electrical conductivity of aqueous solutions, apparently cannot be applied in its present form to explain the conductivity in non-aqueons solutions. It further appears that the auxiliary theories which are based upon the specific inductive capacity, 
494 Electrical Conductizity of Non-aqueous Solutions

the polymerization of the molecules, and the self-ionization of the solvent, are not sufficient to explain satisfactorily the facts that have been accumulated. Notwithstanding the work that has been done, the data at liand are as yet insufficient for the formulation of a theory by means of which a satisfactory explanation can be given of the phenomenon of electrical conductivity of solutions in general.

This investigation was carried on under the supervision of Professor Kahlenberg, to whom I an under 1nany obligations for his valuable suggestions, and I take this means of acknowledging the same.

Taboratory of Physical Chenistry, Cnizersity of Wisconsin, Madison, July 15. Sig9. 